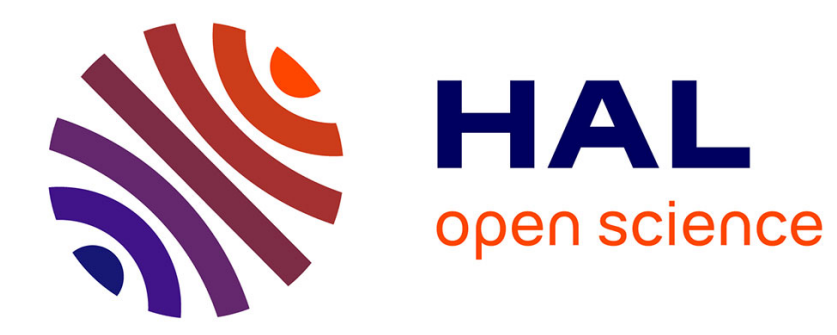

\title{
Error Estimates for a Numerical Scheme for Ferromagnetic Problems
}

Peter Monk, Olivier Vacus

\section{To cite this version:}

Peter Monk, Olivier Vacus. Error Estimates for a Numerical Scheme for Ferromagnetic Problems. [Research Report] RR-3169, INRIA. 1997. inria-00073519

\section{HAL Id: inria-00073519 https://hal.inria.fr/inria-00073519}

Submitted on 24 May 2006

HAL is a multi-disciplinary open access archive for the deposit and dissemination of scientific research documents, whether they are published or not. The documents may come from teaching and research institutions in France or abroad, or from public or private research centers.
L'archive ouverte pluridisciplinaire HAL, est destinée au dépôt et à la diffusion de documents scientifiques de niveau recherche, publiés ou non, émanant des établissements d'enseignement et de recherche français ou étrangers, des laboratoires publics ou privés. 


\section{Error estimates for a numerical scheme for ferromagnetic problems}

Peter Monk, Olivier Vacus

$\mathbf{N}^{\circ} 3169$

Mai 1997

THÈME 4 



\title{
Error estimates for a numerical scheme for ferromagnetic problems
}

\author{
Peter Monk*, Olivier Vacus ${ }^{\dagger}$ \\ Thème 4 - Simulation et optimisation \\ de systèmes complexes \\ Projet Ondes
}

Rapport de recherche $\mathrm{n}^{\circ} 3169$ - Mai 1997 - 30 pages

\begin{abstract}
We propose a finite element method for approximating the non-linear equations describing the electromagnetic field in a ferromagnetic material. Using energy arguments, we prove an optimal convergence rate for the method assuming a sufficiently smooth electromagnetic field. We also verify that the finite element solution satisfies various conservation conditions appropriate to the continuous problem. Finally, we provide some numerical examples.
\end{abstract}

Key-words: Finite element method - Error estimates - Non linear hyperbolic problems

(Résumé : tsvp)

* Department of Mathematical Sciences, University of Delaware, Newark DE 19716, USA

$\dagger$ INRIA, Domaine de Voluceau, B.P.105 78153 Le Chesnay cedex, France.

Unité de recherche INRIA Rocquencourt

Domaine de Voluceau, Rocquencourt, BP 105, 78153 LE CHESNAY Cedex (France) 


\section{Schémas numériques et estimations d'erreur pour le ferromagnétisme}

Résumé : Nous proposons dans ce rapport une méthode de type éléments finis adaptée au problème du ferromagnétisme. Ce problème résulte du couplage des équations de Maxwell instationnaires et de la loi non linéaire dite de Landau-Lifschitz-Gilbert. En nous appuyant sur de la décroissance de certaines énergies, nous montrons la convergence de la méthode pour des champs électromagnétiques suffisamment réguliers. Nous vérifions également que les familles d'éléments finis que nous avons construites respectent les principales propriétés du problème continu. Quelques exemples numériques concluent ce rapport.

Mots-clé : Equations de Maxwell - loi de Landau-Lifchitz-Gilbert - éléments finis - estimations d'erreur. 


\section{Contents}

1 Introduction $\quad 4$

$\begin{array}{llr}2 & \text { Preliminaries } & 6\end{array}$

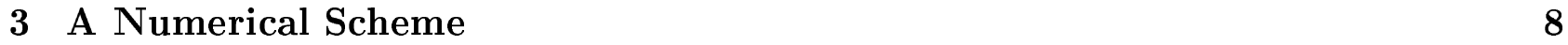

3.1 Tetrahedral elements . . . . . . . . . . . . . . . . 9

3.1.1 Linear Tetrahedral Elements . . . . . . . . . . . . . . 9

3.1.2 Quadratic Tetrahedral Elements . . . . . . . . . . . 10

3.2 Hexahedral elements . . . . . . . . . . . . . . . . . 11

3.2.1 Linear Hexahedral Elements . . . . . . . . . . . . . . . . . . 12

3.2.2 Quadratic Hexahedral Elements . . . . . . . . . . . . 13

3.3 General Comments on Suitable Finite Elements . . . . . . . . . . . . . . 13

4 Conservation Properties of the Semi-discrete Scheme 14

5 Error Estimates $\quad 15$

6 Fully Discrete Scheme $r$

7 Numerical Results $\quad 21$

8 Conclusion. $\quad 23$

$\begin{array}{lr}\text { A A geometrical lemma } & \mathbf{2 6}\end{array}$ 


\section{Introduction}

In order to model the electromagnetic behavior of a ferromagnetic material, the basic Maxwell system must be augmented by an equation describing the influence of the ferromagnet. We suppose that the ferromagnetic material occupies a bounded domain $\Omega \subset \mathbb{R}^{3}$. The electromagnetic field in $\Omega$ is described by four vector functions of position and time: $\boldsymbol{E}$, the electric field, $\boldsymbol{H}$ the magnetic field, $\boldsymbol{B}$ the magnetic induction and $\boldsymbol{M}$ the magnetization. The magnetic variables are related as follows:

$$
\boldsymbol{B}=\mu_{0}(\boldsymbol{H}+\boldsymbol{M})
$$

where $\mu_{0}$ is the magnetic permeability of free space.

The standard Maxwell equations are satisfied throughout $\Omega$ so that

$$
\begin{aligned}
\epsilon_{0} \frac{\partial \boldsymbol{E}}{\partial t}-\nabla \times \boldsymbol{H} & =-\boldsymbol{J} \\
\frac{\partial \boldsymbol{B}}{\partial t}+\nabla \times \boldsymbol{E} & =0 .
\end{aligned}
$$

Here $\epsilon_{0}$ is the permeability of free space and $\boldsymbol{J}$ is the current density. We shall assume a non-conducting ferromagnet, but a conducting ferromagnet can also be modeled by adding an Ohms law term. This does not complicate the numerical algorithm (variable $\epsilon$ is also simple to implement).

To complete the system of equations, we need an equation for the magnetization $\boldsymbol{M}$. This is provided by the Landau-Lifchitz-Gilbert equation (denoted LLG in this paper). This states that

$$
\frac{\partial \boldsymbol{M}}{\partial t}=\frac{|\gamma|}{1+\alpha^{2}}\left(\boldsymbol{H}_{T}(\boldsymbol{H}, \boldsymbol{M}) \times \boldsymbol{M}+\alpha \frac{\boldsymbol{M}}{|\boldsymbol{M}|} \times\left(\boldsymbol{H}_{T}(\boldsymbol{H}, \boldsymbol{M}) \times \boldsymbol{M}\right)\right),
$$

where $\gamma$ is the gyromagnetic factor, $\alpha$ is the damping constant, and $\boldsymbol{H}_{T}(\boldsymbol{H}, \boldsymbol{M})$ is the total magnetic field in the ferromagnet. This total field arises from a number of contributions. The magnetic field is augmented by a given static field $\boldsymbol{H}_{s}$. However ferromagnets also have an anisotropic behavior. There is a preferred direction, called the easy axis, in the direction of a vector $\boldsymbol{p}$. Let $P(\boldsymbol{M})$ denote the projection of $\boldsymbol{M}$ on the plane perpendicular to $\boldsymbol{p}$ so that

$$
P(\boldsymbol{M})=\boldsymbol{M}-(\boldsymbol{p} \cdot \boldsymbol{M}) \boldsymbol{p} .
$$

Then the field of anisotropy is proportional to $P(\boldsymbol{M})$. Thus

$$
\boldsymbol{H}_{T}(\boldsymbol{H}, \boldsymbol{M})=\boldsymbol{H}+\boldsymbol{H}_{s}-K P(\boldsymbol{M})
$$

where $K$ is a constant characterizing the material. In this paper we shall assume $K$ to be independent of position, but in reality $K$ might depend on position. We neglect one further contribution to the total field, called the exchange field. For the applications we have in mind which are mainly modeling radar absorbing materials for stealth applications, this field is not important. Examples of materials of this type are ferrites or garnets which are used in the microwave domain as shifters or circulators for example. For a more complete discussion of the model, see for example $[1,8]$, and for a models including the exchange field see [20].

An important consequence of the LLG equation is that the the norm of $\boldsymbol{M}$ is independent of time. To see this, at least formally, we take the dot product of the LLG equation (4) with $M$ and conclude that

$$
\frac{1}{2} \frac{\partial}{\partial t}|\boldsymbol{M}(\boldsymbol{x}, t)|^{2}=\frac{\partial \boldsymbol{M}}{\partial t}(\boldsymbol{x}, t) \cdot \boldsymbol{M}(\boldsymbol{x}, t)=0
$$


Thus, for almost every $\boldsymbol{x} \in \Omega$, we have the following conservation of the norm of $\boldsymbol{M}$ :

$$
|\boldsymbol{M}(\boldsymbol{x}, t)|=|\boldsymbol{M}(\boldsymbol{x}, 0)|
$$

Using the equations (1), (2), (3) and (4) and eliminating $\boldsymbol{B}$ gives us the following system which we call the Maxwell-LLG system. The electomagnetic field $(\boldsymbol{E}, \boldsymbol{H}, \boldsymbol{M})$ satisfies

$$
\begin{aligned}
\epsilon_{0} \frac{\partial \boldsymbol{E}}{\partial t}-\nabla \times \boldsymbol{H} & =-\boldsymbol{J} \\
\mu_{0} \frac{\partial \boldsymbol{H}}{\partial t}+\nabla \times \boldsymbol{E} & =-\mu_{0} \frac{\partial \boldsymbol{M}}{\partial t} \\
\frac{\partial \boldsymbol{M}}{\partial t} & =\frac{\gamma}{1+\alpha^{2}}\left(\boldsymbol{H}_{T}(\boldsymbol{H}, \boldsymbol{M}) \times \boldsymbol{M}+\alpha \frac{\boldsymbol{M}}{|\boldsymbol{M}|} \times\left(\boldsymbol{H}_{T}(\boldsymbol{H}, \boldsymbol{M}) \times \boldsymbol{M}\right)\right)
\end{aligned}
$$

in $\Omega$ where

$$
\boldsymbol{H}_{T}(\boldsymbol{H}, \boldsymbol{M})=\boldsymbol{H}+\boldsymbol{H}_{s}-K P(\boldsymbol{M}) .
$$

For simplicity, we shall assume that the boundary of $\Omega$ is perfectly conducting. In fact, when we discuss numerical results, we will show how to incorporate impedance boundary conditions. Thus

$$
\boldsymbol{\nu} \times \boldsymbol{E}=0 \text { on } \Gamma=\partial \Omega
$$

where $\boldsymbol{\nu}$ is the unit outward normal to $\Omega$.

Finally, we assume that $\boldsymbol{E}, \boldsymbol{H}$ and $\boldsymbol{M}$ are specified at time $t=0$. Thus

$$
\boldsymbol{E}(t=0)=\boldsymbol{E}_{0}, \quad \boldsymbol{H}(t=0)=\boldsymbol{H}_{0}, \text { and } \boldsymbol{M}(t=0)=\boldsymbol{M}_{0}
$$

where $\boldsymbol{E}_{0}, \boldsymbol{H}_{0}$ and $\boldsymbol{M}_{0}$ are given functions. For physical reasons, these initial fields must satisfy the constraint that

$$
\nabla \cdot\left(\boldsymbol{H}_{0}+\boldsymbol{M}_{0}\right)=0 \text { in } \Omega \text { and } \boldsymbol{\nu} \cdot\left(\boldsymbol{H}_{0}+\boldsymbol{M}_{0}\right)=0 \text { on } \Gamma .
$$

This ensures that $\boldsymbol{B}$ is divergence free.

In this model, the ferromagnet occupies exactly the region of space where $\boldsymbol{M} \neq 0$. So, because of the pointwise conservation of the norm of $\boldsymbol{M}$, the extent of the ferromagnet is determined by the initial distribution of magnetization.

The Maxwell-LLG system has been studied by Joly and Vacus [5, 7, 6] who have established existence and uniqueness for the system in a variety of settings, and have analyzed linearizations of the Maxwell-LLG system. In [5], Joly and Vacus show how a finite difference scheme can be used to compute a solution of the Maxwell-LLG system in 1D, and they prove convergence of the scheme. This scheme can also used in 2D and 3D [6]. Apart from this work, all the work on discretizing the Maxwell-LLG system has involved special cases $(\alpha=0,[10,18,21,3])$, linearization or saturation assumptions $[9,10,18,17,16,3,18,10])$. In this paper we will show how to construct $2 \mathrm{D}$ and $3 \mathrm{D}$ finite element methods for the full problem. We shall also provide some preliminary analysis of the method. The analysis is based on the work of Monk [11], but the analysis here is more difficult since the problem is non-linear. In particular we show how a certain class of finite element methods can be used to approximate the Maxwell-LLG equations while preserving energy decay and the norm of $\boldsymbol{M}$. This allows us to prove error estimates.

$\mathrm{RR} \mathrm{n}^{\circ} 3169$ 


\section{Preliminaries}

In order to write a variational problem suitable for finite element discretization we need some notation and function spaces. Let

$$
(\boldsymbol{u}, \boldsymbol{v})=\int_{\Omega} \boldsymbol{u} \cdot \boldsymbol{v} d V
$$

and $\|\boldsymbol{u}\|=\sqrt{(\boldsymbol{u}, \boldsymbol{u})}$. We use the usual notation for Sobolev spaces of scalar function so that $H^{s}(\Omega)$ denotes the space of functions with $s$ square integrable derivatives on $\Omega$ equipped with the norm $\|.\|_{s}$ (so $\left.\|.\|_{0}=\|\|.\right)$. In addition, recall that

$$
H_{0}(\operatorname{curl} ; \Omega)=\left\{\boldsymbol{u} \in\left(L_{2}(\Omega)\right)^{3} \mid \nabla \times \boldsymbol{u} \in\left(L_{2}(\Omega)\right)^{3} \text { and } \boldsymbol{\nu} \times \boldsymbol{u}=0 \text { on } \Gamma\right\} .
$$

In order to simplify notation we denote by $F(\boldsymbol{H}, \boldsymbol{M})$ the right hand side of (7) so that

$$
F(\boldsymbol{H}, \boldsymbol{M})=\frac{\gamma}{1+\alpha^{2}}\left(\boldsymbol{H}_{T}(\boldsymbol{H}, \boldsymbol{M}) \times \boldsymbol{M}+\alpha \frac{\boldsymbol{M}}{|\boldsymbol{M}|} \times\left(\boldsymbol{H}_{T}(\boldsymbol{H}, \boldsymbol{M}) \times \boldsymbol{M}\right) .\right)
$$

To obtain a variational scheme we proceed formally to multiply (6), (7) and (8) by test functions and integrate over $\Omega$. Then integrating the curl term in (6) by parts and using the boundary data (10) we obtain a suitable problem. In particular, for a given final time $T>0$, we seek

$$
\begin{aligned}
\boldsymbol{E} & \in C\left(0, T ; H_{0}(\operatorname{curl} ; \Omega)\right) \cap C^{1}\left(0, T ;\left(L_{2}(\Omega)\right)^{3}\right) \\
\boldsymbol{H} & \in C^{1}\left(0, T ;\left(L_{2}(\Omega)\right)^{3}\right) \\
\boldsymbol{M} & \in C^{1}\left(0, T ;\left(L_{2}(\Omega)\right)^{3}\right)
\end{aligned}
$$

such that

$$
\begin{aligned}
\left(\frac{\partial}{\partial t} \boldsymbol{E}, \boldsymbol{\psi}\right)-(\boldsymbol{H}, \nabla \times \boldsymbol{\psi}) & =(\boldsymbol{J}, \boldsymbol{\psi}) \quad \forall \boldsymbol{\psi} \in H_{0}(\operatorname{curl} ; \Omega) \\
\left(\frac{\partial}{\partial t} \boldsymbol{H}, \boldsymbol{\phi}\right)+(\nabla \times \boldsymbol{E}, \boldsymbol{\phi}) & =-\left(\frac{\partial}{\partial t} \boldsymbol{M}, \boldsymbol{\phi}\right) \quad \forall \boldsymbol{\phi} \in\left(L_{2}(\Omega)\right)^{3} \\
\left(\frac{\partial}{\partial t} \boldsymbol{M}, \boldsymbol{\xi}\right) & =(F(\boldsymbol{H}, \boldsymbol{M}), \boldsymbol{\xi}) \quad \forall \boldsymbol{\xi} \in\left(L_{2}(\Omega)\right)^{3}
\end{aligned}
$$

subject to the initial conditions in (11). As mentioned in the introduction, the existence and uniqueness of solutions to the 1D Maxwell-LLG equation has been studied by Joly and Vacus. Here we shall simply assume the existence of a unique solution to the above variational problem (we shall prove uniqueness shortly) in the indicated spaces. Later, when we prove error estimates, we shall make further smoothness assumptions on the solution sufficient for error estimates to hold.

The error analysis of the finite element method is based on a simple continuous dependence result for the Maxwell-LLG equations. Next we prove this result under the assumption that the Maxwell-LLG equations have a unique and bounded solution. Suppose that $\left(\boldsymbol{E}_{1}, \boldsymbol{H}_{1}, \boldsymbol{M}_{1}\right)$ and $\left(\boldsymbol{E}_{2}, \boldsymbol{H}_{2}, \boldsymbol{M}_{2}\right)$ are two solutions of the Maxwell-LLG equations. Let

$$
\begin{aligned}
\boldsymbol{e} & =\boldsymbol{E}_{2}-\boldsymbol{E}_{1} \\
\boldsymbol{h} & =\boldsymbol{H}_{2}-\boldsymbol{H}_{1} \\
\boldsymbol{m} & =\boldsymbol{M}_{2}-\boldsymbol{M}_{1} .
\end{aligned}
$$


Then we define

$$
E(t)=\|\boldsymbol{e}\|^{2}+\|\boldsymbol{h}\|^{2}+\|\boldsymbol{m}\|^{2} .
$$

We prove the following result:

Theorem 2.1 Suppose $\boldsymbol{H}_{i}$ and $\boldsymbol{M}_{i}, i=1,2$ are bounded functions in space and time. Then, for $0 \leq t \leq T$,

$$
E(t) \leq E(0) \exp (\mu t)
$$

where

$$
\mu=\frac{2 \gamma(1+\alpha)}{1+\alpha^{2}}\left(\left\|\boldsymbol{H}_{s}\right\|_{\infty, \Omega_{T}}+\left\|\boldsymbol{M}_{1}\right\|_{\infty, \Omega_{T}}+\left\|\boldsymbol{M}_{2}\right\|_{\infty, \Omega_{T}}+\left\|\boldsymbol{H}_{2}\right\|_{\infty, \Omega_{T}}\right)
$$

Remark: Note that we have used a constant $\mu$ that does not depend symmetrically on $\boldsymbol{M}_{i}$ and $\boldsymbol{H}_{i}, i=1,2$. This allows us to obtain a discrete version of this result in which the corresponding quantity is bounded independent of the mesh.

To prove Theorem 2.1, as well as the error estimates later in the paper, start by proving the following the following geometrical lemma concerning $F$ (defined in (12):

Lemma 2.1 For all vectors $\left(\boldsymbol{H}_{s}, \boldsymbol{H}_{1}, \boldsymbol{H}_{2}, \boldsymbol{M}_{1}, \boldsymbol{M}_{2}\right) \in\left(\mathbb{R}^{3}\right)^{4}$, we have, with $\gamma^{\prime}=\frac{\gamma(1+\alpha)}{1+\alpha^{2}}$,

$$
\begin{aligned}
& \left|F\left(\boldsymbol{H}_{2}, \boldsymbol{M}_{2}\right)-F\left(\boldsymbol{H}_{1}, \boldsymbol{M}_{1}\right)\right| \\
& \quad \leq \gamma^{\prime}\left(\left|\boldsymbol{H}_{s}\right|+\left|\boldsymbol{M}_{1}\right|+\left|\boldsymbol{M}_{2}\right|+\left|\boldsymbol{H}_{2}\right|\right)\left(\left|\boldsymbol{M}_{2}-\boldsymbol{M}_{1}\right|+\left|\boldsymbol{H}_{2}-\boldsymbol{H}_{1}\right|\right)
\end{aligned}
$$

Proof. Since

$$
\boldsymbol{H}_{T 2} \times \boldsymbol{M}_{\mathbf{2}}-\boldsymbol{H}_{T 1} \times \boldsymbol{M}_{\mathbf{1}}=\boldsymbol{H}_{T 2} \times\left(\boldsymbol{M}_{\mathbf{2}}-\boldsymbol{M}_{\mathbf{1}}\right)+\left(\boldsymbol{H}_{T 2}-\boldsymbol{H}_{T 1}\right)
$$

we have

$$
\left|\boldsymbol{H}_{T 2} \times \boldsymbol{M}_{\mathbf{2}}-\boldsymbol{H}_{T 1} \times \boldsymbol{M}_{\mathbf{1}}\right| \leq\left|\boldsymbol{H}_{2}\right|\left|\boldsymbol{M}_{2}-\boldsymbol{M}_{1}\right|+\left|\boldsymbol{M}_{1}\right|\left|\boldsymbol{H}_{T 2}-\boldsymbol{H}_{T 1}\right|
$$

and the reader can easily see that the main point of the lemma is then to prove that

$$
\left|\frac{\boldsymbol{M}_{\mathbf{2}}}{\left|\boldsymbol{M}_{\mathbf{2}}\right|} \times\left(\boldsymbol{H}_{T 2} \times \boldsymbol{M}_{\mathbf{2}}\right)-\frac{\boldsymbol{M}_{\mathbf{1}}}{\left|\boldsymbol{M}_{\mathbf{1}}\right|} \times\left(\boldsymbol{H}_{T 1} \times \boldsymbol{M}_{\mathbf{1}}\right)\right| \leq\left|\boldsymbol{H}_{2}\right|\left|\boldsymbol{M}_{2}-\boldsymbol{M}_{1}\right|+\left|\boldsymbol{M}_{1}\right|\left|\boldsymbol{H}_{T 2}-\boldsymbol{H}_{T 1}\right|
$$

This is done in appendix at the end of this paper.

Finally we conclude the estimate by noting that

$$
\left|\boldsymbol{H}_{T}(\boldsymbol{H}, \boldsymbol{M})\right| \leq|\boldsymbol{H}|+\left|\boldsymbol{H}_{s}\right|+|\boldsymbol{M}|
$$

and

$$
\left|\boldsymbol{H}_{T}\left(\boldsymbol{H}_{1}, \boldsymbol{M}_{1}\right)-\boldsymbol{H}_{T}\left(\boldsymbol{H}_{2}, \boldsymbol{M}_{2}\right)\right| \leq\left|\mathbf{H}_{1}-\mathbf{H}_{2}\right|+\left|\mathbf{M}_{1}-\mathbf{M}_{2}\right| .
$$

¿From this argument, we see that we could also take as Lipschitz constant

$$
C\left(\boldsymbol{H}_{s}, \boldsymbol{H}_{2}, \boldsymbol{M}_{1}, \boldsymbol{M}_{2}\right)=\frac{\gamma(1+\alpha)}{1+\alpha^{2}} \max \left\{\left|\boldsymbol{M}_{1}\right|,\left|\boldsymbol{H}_{2}\right|+\left|\boldsymbol{H}_{s}\right|+\left|\boldsymbol{M}_{2}\right|\right\}
$$

and that $\frac{\gamma(1+\alpha)}{1+\alpha^{2}}\left(\left|\boldsymbol{H}_{s}\right|+\left|\boldsymbol{M}_{1}\right|+\left|\boldsymbol{M}_{2}\right|+\left|\boldsymbol{H}_{2}\right|\right)$ is also a possible choice.

$\mathrm{RR} \mathrm{n}^{\circ} 3169$ 
Proof. [of Theorem 2.1]. We note that by the linearity of (6) and (7)

$$
\begin{aligned}
\frac{1}{2} \frac{d}{d t}\left(\|\boldsymbol{e}\|^{2}+\|\boldsymbol{h}\|^{2}\right) & =\left(\boldsymbol{e}_{t}, \boldsymbol{e}\right)-(\boldsymbol{h}, \nabla \times \boldsymbol{e})+\left(\boldsymbol{h}_{t}, \boldsymbol{h}\right)+(\nabla \times \boldsymbol{e}, \boldsymbol{h}) \\
& =-\left(\boldsymbol{m}_{t}, \boldsymbol{h}\right)
\end{aligned}
$$

In addition

$$
\frac{1}{2} \frac{d}{d t}\|\boldsymbol{m}\|^{2}=\left(\boldsymbol{m}_{t}, \boldsymbol{m}\right)
$$

Hence

$$
\frac{1}{2} \frac{d}{d t}\left(\|\boldsymbol{e}\|^{2}+\|\boldsymbol{h}\|^{2}+\|\boldsymbol{m}\|^{2}\right)=-\left(\boldsymbol{m}_{t}, \boldsymbol{h}\right)+\left(\boldsymbol{m}_{t}, \boldsymbol{m}\right)=\left(\boldsymbol{m}_{t}, \boldsymbol{m}-\boldsymbol{h}\right)
$$

where

$$
\boldsymbol{m}_{t}=F\left(\boldsymbol{H}_{\mathbf{2}}, \boldsymbol{M}_{\mathbf{2}}\right)-F\left(\boldsymbol{H}_{\mathbf{1}}, \boldsymbol{M}_{\mathbf{1}}\right) .
$$

Integrating (18) in time and using the Cauchy-Schwarz inequality and Lemma 2.1, we have the following estimate:

$$
\begin{aligned}
E(t)-E(0) \leq & \int_{0}^{t}\left(F\left(\boldsymbol{H}_{\mathbf{2}}, \boldsymbol{M}_{\mathbf{2}}\right)-F\left(\boldsymbol{H}_{\mathbf{1}}, \boldsymbol{M}_{\mathbf{1}}\right), \boldsymbol{m}-\boldsymbol{h}\right) d t \\
\leq & \int_{0}^{t}\left\|F\left(\boldsymbol{H}_{\mathbf{2}}, \boldsymbol{M}_{\mathbf{2}}\right)-F\left(\boldsymbol{H}_{\mathbf{1}}, \boldsymbol{M}_{\mathbf{1}}\right)\right\|(\|\boldsymbol{h}\|+\|\boldsymbol{m}\|) d t \\
\leq & \frac{\gamma(1+\alpha)}{1+\alpha^{2}}\left(\left\|\boldsymbol{H}_{s}\right\|_{\infty, \Omega_{T}}+\left\|\boldsymbol{M}_{1}\right\|_{\infty, \Omega_{T}}+\left\|\boldsymbol{M}_{2}\right\|_{\infty, \Omega_{T}}\right. \\
& \left.+\left\|\boldsymbol{H}_{2}\right\|_{\infty, \Omega_{T}}\right) \int_{0}^{t}(\|\boldsymbol{h}\|+\|\boldsymbol{m}\|)^{2} d t .
\end{aligned}
$$

This may be written

$$
E(t) \leq E(0)+\mu \int_{0}^{t} E(s) d s .
$$

An application of Gronwall's inequality proves the lemma.

\section{A Numerical Scheme}

The main point of this paper is to propose and analyze some finite element schemes for approximating the Maxwell-LLG equations. We start by describing a variety of different finite elements that fit into the general framework we use. Let $\tau_{h}$ be a mesh of $\Omega$ of regular and quasi-uniform elements of maximum diameter $h$. At this stage the mesh could either consist of tetrahedral or hexahedral elements.

Using the mesh $\tau_{h}$, we will construct finite element spaces $U_{h} \subset H_{0}$ (curl; $\Omega$ ) and $V_{h} \subset$ $\left(L_{2}(\Omega)\right)^{3} \cap\left(L_{\infty}(\Omega)\right)^{3}$ such that $\nabla \times U_{h} \subset V_{h}$. Assuming we can construct suitable spaces $U_{h}$ and $V_{h}$, the numerical method we shall analyze is to find $\left(\boldsymbol{E}_{h}, \boldsymbol{H}_{h}, \boldsymbol{M}_{h}\right) \in U_{h} \times V_{h} \times V_{h}$ such that

$$
\begin{aligned}
\left(\frac{\partial}{\partial t} \boldsymbol{E}_{h}, \boldsymbol{\psi}_{h}\right)-\left(\boldsymbol{H}_{h}, \nabla \times \boldsymbol{\psi}_{h}\right) & =\left(\boldsymbol{J}, \boldsymbol{\psi}_{h}\right) \quad \forall \boldsymbol{\psi}_{h} \in U_{h} \\
\left(\frac{\partial}{\partial t} \boldsymbol{H}_{h}, \boldsymbol{\phi}_{h}\right)+\left(\nabla \times \boldsymbol{E}_{h}, \boldsymbol{\phi}_{h}\right) & =-\left(\frac{\partial}{\partial t} \boldsymbol{M}_{h}, \boldsymbol{\phi}_{h}\right) \quad \forall \boldsymbol{\phi}_{h} \in V_{h} \\
\left(\frac{\partial}{\partial t} \boldsymbol{M}_{h}, \boldsymbol{\xi}_{h}\right)_{h} & =\left(F\left(\boldsymbol{H}_{h}, \boldsymbol{M}_{h}\right), \boldsymbol{\xi}_{h}\right)_{h} \quad \boldsymbol{\xi}_{h} \in V_{h}
\end{aligned}
$$


where $(\cdot, \cdot)_{h}$ is a suitable discrete inner product depending on the space $V_{h}$.

\subsection{Tetrahedral elements}

If the mesh is made of tetrahedra, we propose to use edge elements to approximate the electric field, and discontinuous elements for the magnetic field and magnetization. We shall describe two suitable elements based on the Mur-Nédélec family [13, 15]. The first element is described in some detail since it is the one we use in our computations and we also need to define some notation for the upcoming error estimate. Let $P_{k}$ denote the space of polynomials in $x, y$ and $z$ of total degree at most $k$.

\subsubsection{Linear Tetrahedral Elements}

We approximate the electric field by piecewise linear polynomials so that

$$
U_{h}=\left\{\boldsymbol{u} \in H_{0}(\operatorname{curl} ; \Omega) \mid \boldsymbol{u} \in\left(P_{1}\right)^{3}\right\} \text {. }
$$

The degrees of freedom for this space are the average value and first moment of the tangential component of the electric field along each edge in the mesh (see [14] and Figure 1). Thus if $K$ is a tetrahedron in the mesh, the degrees of freedom are

$$
\int_{e}\left(\boldsymbol{u}_{h} \cdot \boldsymbol{\tau}_{e}\right) d s \text { and } \int_{e}\left(\boldsymbol{u}_{h} \cdot \boldsymbol{\tau}_{e}\right) s d s
$$

where $e$ is an edge of $K$ with unit tangent $\boldsymbol{\tau}_{e}$ and $s$ is arc length along $e$. These degrees of freedom can be used to define an interpolation operator $r_{h}:\left(W^{1, s}(\Omega)\right)^{3} \rightarrow U_{h}, s>2$ such that

$$
\left\|\boldsymbol{u}-r_{h} \boldsymbol{u}\right\|+\left\|\nabla \times\left(\boldsymbol{u}-r_{h} \boldsymbol{u}\right)\right\| \leq C h^{p} \mid\|\boldsymbol{u}\|_{p+1}
$$

where $p=1$ and

$$
\|\boldsymbol{u}\|_{p+1}=\sqrt{\sum_{K \in \tau_{h}}\|\boldsymbol{u}\|_{p+1, K}^{2}} .
$$

This element can be extended to curvilinear tetrahedra using the method of Dubois [4] (the paper of Dubois is for first kind edge elements [14], which could also be used to discretize the electric field).

For the magnetic variables we use piecewise constants

$$
V_{h}=\left\{\boldsymbol{v} \in\left(L_{2}(\Omega)\right)^{3} \mid \boldsymbol{v} \in\left(P_{0}\right)^{3}\right\} .
$$

The degrees of freedom for this space are the value of the field at the centroid of each element. These degrees of freedom define an interpolation operator

$$
w_{h}^{Q}:\left(H^{2}(\Omega)\right)^{3} \rightarrow V_{h}
$$

such that

$$
\left\|\boldsymbol{v}-w_{h}^{Q} \boldsymbol{v}\left|\leq C h^{p}\right| \mid \boldsymbol{v}\right\|_{p+1}
$$

for $p=2$.

$\mathrm{RR} \mathrm{n}^{\circ} 3169$ 

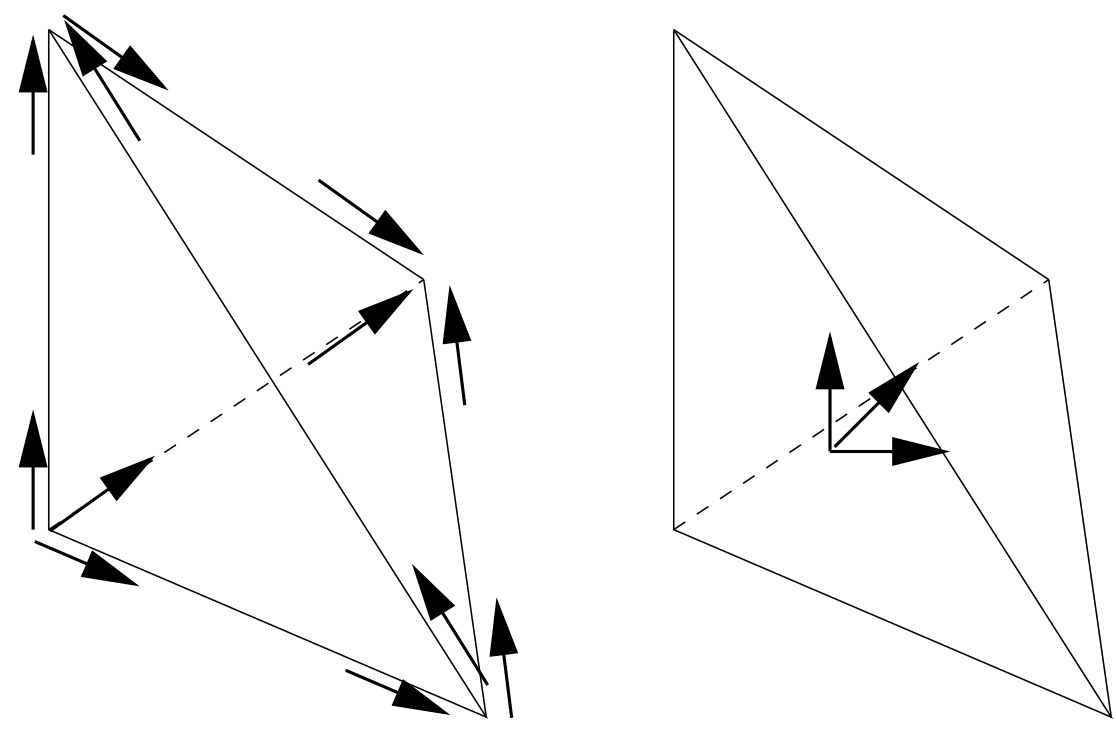

Figure 1: Here we show the degrees of freedom of the linear edge space on tetrahedra (left, used for the electric field) and the degrees of freedom for the piecewise constant space (right used for the magnetic field).

Finally we need to define a suitable discrete inner product. If $\boldsymbol{a}_{K}$ is the centroid of tetrahedron $K$, and if we define the discrete inner product

$$
(\boldsymbol{u}, \boldsymbol{v})_{h}=\sum_{K} \operatorname{vol}(K) \boldsymbol{u}\left(\boldsymbol{a}_{K}\right) \cdot \boldsymbol{v}\left(\boldsymbol{a}_{K}\right)
$$

then this inner product coincides with the exact inner product on $V_{h}$ since the quadrature integrates linear functions exactly so

$$
\left(\boldsymbol{u}_{h}, \boldsymbol{v}\right)_{h}=\left(\boldsymbol{u}_{h}, \boldsymbol{v}_{h}\right) \quad \forall \boldsymbol{u}_{h}, \boldsymbol{v}_{h} \in V_{h} .
$$

We also define the following discrete seminorm on suitably smooth function $\boldsymbol{u}$ :

$$
\|\boldsymbol{u}\| \|=\sqrt{(\boldsymbol{u}, \boldsymbol{u})_{h}}
$$

This is exactly the standard $\left(L_{2}(\Omega)\right)^{3}$ norm for functions in $V_{h}$.

A particular attraction of this element is that it can be partially mass-lumped (see section 7), yet also handle anisotropic material properties.

\subsubsection{Quadratic Tetrahedral Elements}

We approximate the electric field by quadratic polynomials so that

$$
U_{h}=\left\{\boldsymbol{u} \in H_{0}(\operatorname{curl} ; \Omega) \mid \boldsymbol{u} \in\left(P_{2}\right)^{3}\right\} \text {. }
$$

The degrees of freedom for this space are the average value, the first and second moments of the tangential component of the electric field along each edge in the mesh, and suitable moments on each face (see [15] and Figure 2). For this element (23) is satisfied with $p=2$. 

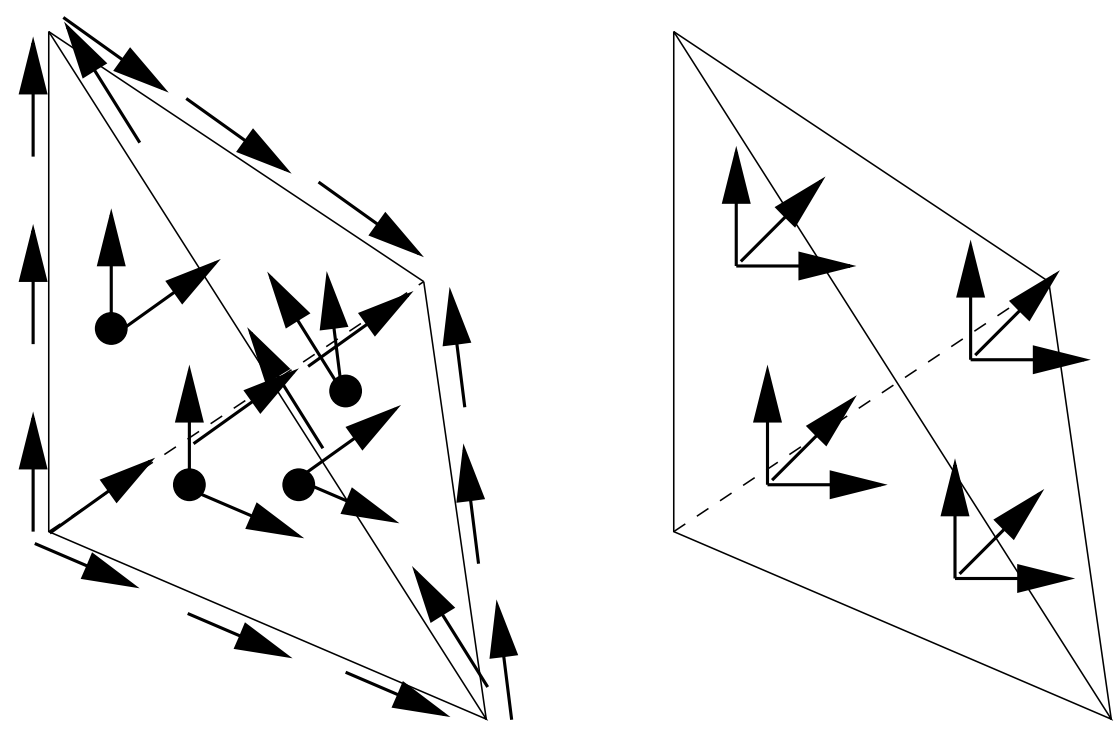

Figure 2: Here we show the degrees of freedom of the quadratic edge space on tetrahedra (left, used for the electric field) and the degrees of freedom for the piecewise linear space (right used for the magnetic field). The solid • signifies a third moment degree of freedom at the appropriate positions.

For the magnetic variables we use piecewise linear

$$
V_{h}=\left\{\boldsymbol{v} \in\left(L_{2}(\Omega)\right)^{3} \mid \boldsymbol{v} \in\left(P_{1}\right)^{3}\right\} .
$$

The degrees of freedom for this space are the value of the field at four points in the element. These are chosen to give an accurate discrete inner product. Let $\boldsymbol{a}_{K}$ be the centroid of element $K$ having vertices $\left\{\boldsymbol{a}_{i}^{K}\right\}_{i=1}^{4}$. Then the four interpolation points are

$$
\boldsymbol{\xi}_{i}^{K}=a \boldsymbol{a}_{K}+(1-a) \boldsymbol{a}_{i}^{K}, \quad 1 \leq i \leq 4,
$$

where $a=(1-\sqrt{5} / 5)$. Using this interpolant, estimate $(24)$ is satisfied with $p=2$.

We use the interpolation points to define the discrete inner product

$$
(\boldsymbol{u}, \boldsymbol{v})_{h}=\sum_{K} \frac{\operatorname{vol}(K)}{4} \sum_{i=1}^{4} \boldsymbol{u}\left(\boldsymbol{\xi}_{i}^{K}\right) \cdot \boldsymbol{v}\left(\boldsymbol{\xi}_{i}^{K}\right) .
$$

This inner product coincides with the exact inner product on $V_{h}$ since the quadrature integrates quadratic functions exactly so

$$
\left(\boldsymbol{u}_{h}, \boldsymbol{v}\right)_{h}=\left(\boldsymbol{u}_{h}, \boldsymbol{v}_{h}\right) \quad \forall \boldsymbol{u}_{h}, \boldsymbol{v}_{h} \in V_{h} .
$$

In comparison to the linear element, this element suffers from the disadvantage that it can not be mass-lumped.

\subsection{Hexahedral elements}

If the mesh is made of hexahedra, we still propose to use edge elements to approximate the electric field, and discontinuous elements for the magnetic field and magnetization. We shall $\mathrm{RR} \mathrm{n}^{\circ} 3169$ 

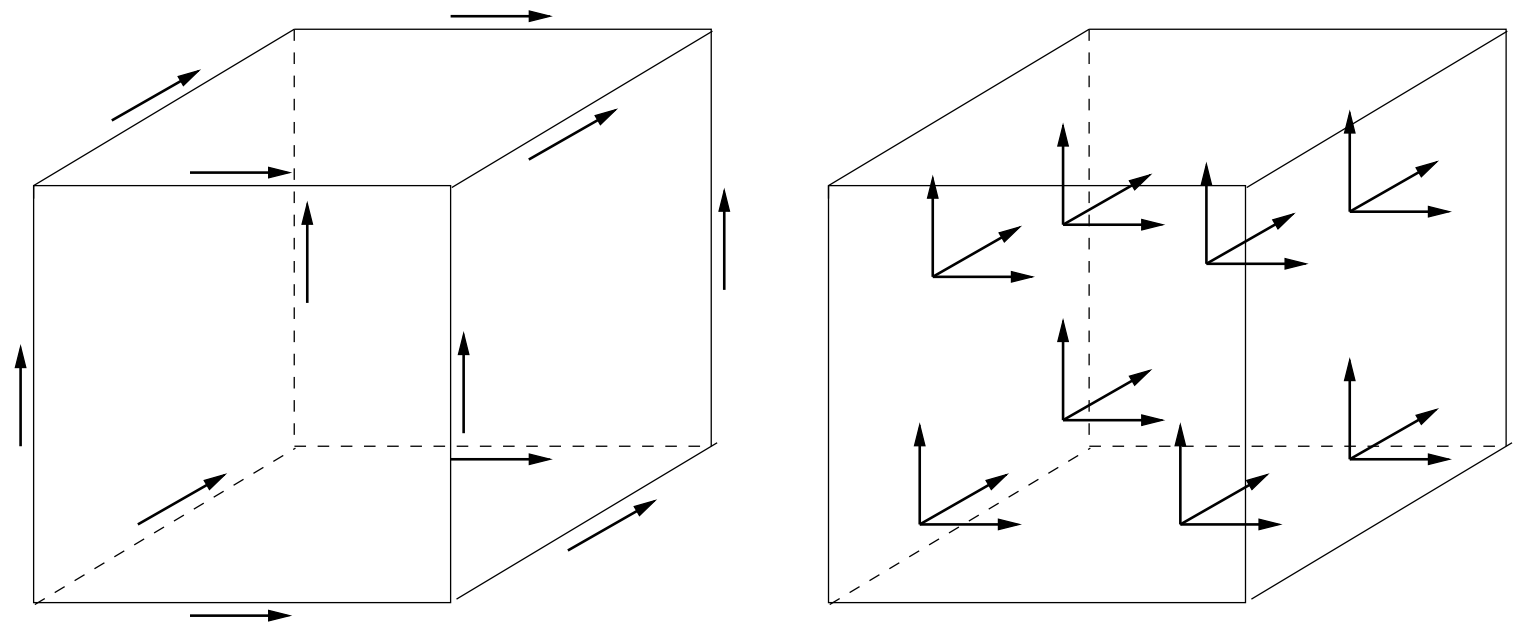

Figure 3: Here we show the degrees of freedom of the linear edge space on hexahedra (left, used for the electric field) and the degrees of freedom for the piecewise linear space (right - used for the magnetic field).

describe two suitable elements based on the Nédélec family [14]. For simplicity, we shall restrict ourselves to right hexahedra with edges parallel to the coordinate axis. Isoparametric elements of this type are also possible (see for example [19] and references therein), although we know of no error analysis for these elements. Let $Q_{k, l, m}$ denote the space of polynomials of degree $k$ in $x, l$ in $y$ and $m$ in $z$.

\subsubsection{Linear Hexahedral Elements}

We approximate the electric field by anisotropic piecewise polynomials:

$$
U_{h}=\left\{\boldsymbol{u} \in H_{0}(\operatorname{curl} ; \Omega) \mid \boldsymbol{u} \in Q_{0,1,1} \times Q_{1,0,1} \times Q_{1,1,0}\right\}
$$

The degrees of freedom for this space are the average value of the tangential component of the electric field on each edge in the mesh (see [14] and Figure 3). Estimate (23) is satisfied with $p=1$.

For the magnetic variables we use piecewise trilinear

$$
V_{h}=\left\{\boldsymbol{v} \in\left(L_{2}(\Omega)\right)^{3} \mid \boldsymbol{v} \in\left(Q_{1,1,1}\right)^{3}\right\}
$$

The degrees of freedom for this space are the value of the field at the two point tensor product Gauss points. Estimate (23) is satisfied with $p=1$ (and with $p=2$ ).

To define the discrete inner product, let $\boldsymbol{\xi}_{i, j, k}^{K} 1 \leq i, j, k \leq 2$ be the tensor product Gauss points in hexahedron $K$, then we define the discrete inner product

$$
(\boldsymbol{u}, \boldsymbol{v})_{h}=\sum_{K} \sum_{i=1}^{2} \sum_{j=1}^{2} \sum_{k=1}^{2} \frac{\operatorname{vol}(K)}{4} \boldsymbol{u}\left(\boldsymbol{\xi}_{i, j, k}^{K}\right) \cdot \boldsymbol{v}\left(\boldsymbol{\xi}_{i, j, k}^{K}\right)
$$

then this inner product coincides with the exact inner product on $V_{h}$ (since the two point Gauss rule is exact for cubic polynomials). 

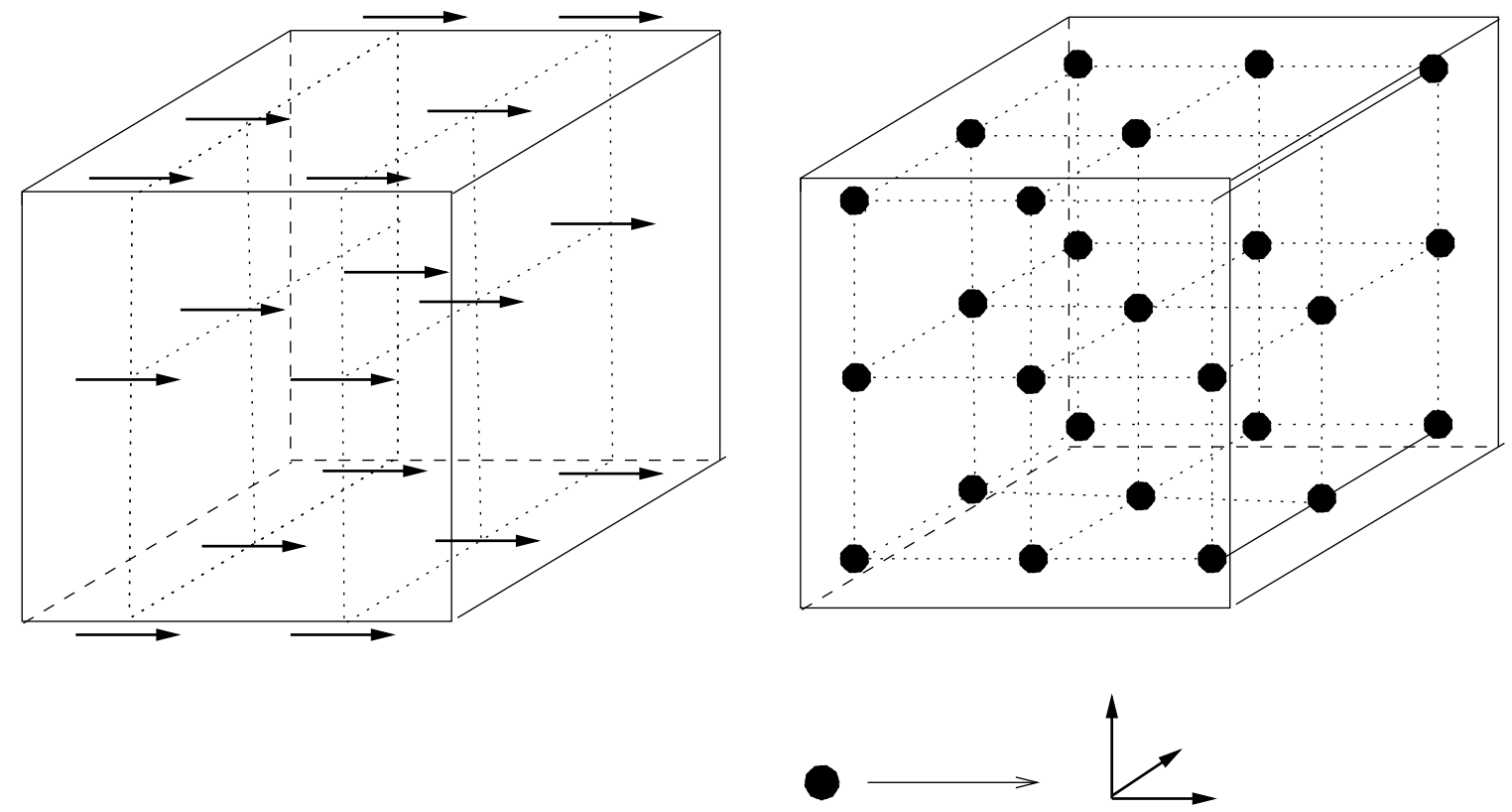

Figure 4: Here we show the degrees of freedom of the quadratic edge space on hexahedra. In the left panel, we show just the $x$ component of the electric field. In the right panel we show the degrees of freedom for the discontinuous piecewise quadratic space used for the magnetic field. Here the $\bullet$ marks the position of the vector degrees of freedom.

\subsubsection{Quadratic Hexahedral Elements}

The linear hexahedral element is the first of a family of elements. Next we describe the quadratic element in this family, and leave higher order elements to the reader. We approximate the electric field by

$$
U_{h}=\left\{\boldsymbol{u} \in H_{0}(\operatorname{curl} ; \Omega) \mid \boldsymbol{u} \in Q_{1,2,2} \times Q_{2,1,2} \times Q_{2,2,1}\right\} .
$$

The degrees of freedom are described in [14] and Figure 4. Estimate (23) is satisfied with $p=2$.

For the magnetic variables we use piecewise tri-quadratic polynomials:

$$
V_{h}=\left\{\boldsymbol{v} \in\left(L_{2}(\Omega)\right)^{3} \mid \boldsymbol{v} \in\left(Q_{2,2,2}\right)^{3}\right\} .
$$

Estimate (24) is satisfied with $p=2$.

To define the discrete inner product, let $\boldsymbol{\xi}_{i, j, k}^{K} 1 \leq i, j, k \leq 3$ be the $3 \times 3$ tensor product Gauss points in hexahedron $K$, then we define the discrete inner product

$$
(\boldsymbol{u}, \boldsymbol{v})_{h}=\sum_{K} \sum_{i=1}^{3} \sum_{j=1}^{3} \sum_{k=1}^{3} w_{i, j, k} \frac{\operatorname{vol}(K)}{4} \boldsymbol{u}\left(\boldsymbol{\xi}_{i, j, k}^{K}\right) \cdot \boldsymbol{v}\left(\boldsymbol{\xi}_{i, j, k}^{K}\right)
$$

where $w_{i, j, k}, 1 \leq i, j, k \leq 3$ are the quadrature weights for the tensor product formula on $[0,1]^{3}$.

\subsection{General Comments on Suitable Finite Elements}

The finite element spaces, and the discrete inner product that we have suggested above have the following properties:

$\mathrm{RR} \mathrm{n}^{\circ} 3169$ 
- $\nabla \times U_{h} \in V_{h}$.

- The discrete inner product is computed using quadrature on each element in the mesh. The quadrature points are assumed to coincide exactly with the degrees of freedom for $V_{h}$.

- The inner product coincides with the $\left(L_{2}(\Omega)\right)^{3}$ inner product on $V_{h}$.

With these properties (and suitable interpolation operators) we can prove the error estimates in the next sections.

Perhaps the most obvious scheme that does not fit into our frame-work is the 3D finite element analogue of the method used in [6]. This corresponds to using the linear edge space on hexahedra, and a piecewise constant space for the magnetic variables. This does not satisfy $\nabla \times U_{h} \subset V_{h}$.

\section{Conservation Properties of the Semi-discrete Scheme}

The numerical scheme conserves the magnitude of the magnetization at the quadrature points of the discrete inner product. This is the discrete analogue of (5).

Lemma 4.1 Let $\boldsymbol{a}_{i}$ be a quadrature point for the integration scheme used to compute the discrete inner product. For each time $t$,

$$
\left|\boldsymbol{M}_{h}\left(\boldsymbol{a}_{i}, t\right)\right|=\left|\boldsymbol{M}_{h}\left(\boldsymbol{a}_{i}, 0\right)\right|
$$

In particular, $\left\|\boldsymbol{M}_{h}\right\|_{\infty, \Omega}$ is bounded independent of $t$ and $h$.

Proof. We choose $\boldsymbol{\xi}_{h} \in V_{h}$ to interpolate $\boldsymbol{M}_{h}$ at $\boldsymbol{a}_{i}$ and interpolate zero at all other quadrature (or interpolation) points. Let $w_{i}$ be the corresponding quadrature weight in the discrete inner product. Using $\boldsymbol{\xi}_{h}$ in $(22)$ we conclude that

$$
\left(\frac{\partial}{\partial t} \boldsymbol{M}_{h}, \boldsymbol{M}_{h}\right)_{h}=w_{i} \frac{\partial}{\partial t} \boldsymbol{M}_{h}\left(\boldsymbol{a}_{i}\right) \cdot \boldsymbol{M}_{h}\left(\boldsymbol{a}_{i}\right)=w_{i} F(\boldsymbol{H}, \boldsymbol{M})\left(\boldsymbol{a}_{i}\right) \cdot \boldsymbol{M}_{h}\left(\boldsymbol{a}_{i}\right)=0 .
$$

The method also conserves the energy of the electromagnetic field, again mimicking the continuous case (see [5]).

Lemma 4.2 Suppose $\boldsymbol{J}=0$, then for each time $t$,

$$
\begin{gathered}
\left\|\boldsymbol{E}_{h}(t)\right\|^{2}+\left\|\boldsymbol{H}_{h}(t)\right\|^{2}+\left\|\left|\left(\boldsymbol{H}_{s}-\boldsymbol{M}_{h}\right)(t)\|\|^{2}+K^{2}\left\|\mid P\left(\boldsymbol{M}_{h}\right)(t)\right\| \|^{2}\right.\right. \\
\leq\left\|\boldsymbol{E}_{h}(0)\right\|^{2}+\left\|\boldsymbol{H}_{h}(0)\right\|^{2}+\|\|\left(\boldsymbol{H}_{s}-\boldsymbol{M}_{h}\right)(0)\|\|^{2}+K^{2}\|\| P\left(\boldsymbol{M}_{h}\right)(0)\|\|^{2} .
\end{gathered}
$$

Proof. The proof consists in checking that the energy decay proof in [5] holds for the finite element problem. Recall that we assume $\boldsymbol{J}=0$. Selecting $\boldsymbol{\psi}_{h}=\boldsymbol{E}_{h}$ and $\boldsymbol{\phi}_{h}=\boldsymbol{H}_{h}$ in (20) and (21) and adding the resulting equations we obtain

$$
\begin{aligned}
\left(\frac{\partial}{\partial t} \boldsymbol{E}_{h}, \boldsymbol{E}_{h}\right)+\left(\frac{\partial}{\partial t} \boldsymbol{H}_{h}, \boldsymbol{H}_{h}\right) & =-\left(\frac{\partial}{\partial t} \boldsymbol{M}_{h}, \boldsymbol{H}_{h}\right) \\
& =-\left(\frac{\partial}{\partial t} \boldsymbol{M}_{h}, \boldsymbol{H}_{h}\right)_{h} \\
& =-\left(\frac{\partial}{\partial t} \boldsymbol{M}_{h}, \boldsymbol{H}_{h}+\boldsymbol{H}_{e f f}\right)_{h}+\left(\frac{\partial}{\partial t} \boldsymbol{M}_{h}, \boldsymbol{H}_{e f f}\right)_{h}
\end{aligned}
$$


where we have used the fact that the discrete inner product is exact on $V_{h} \times V_{h}$, and the effective field is defined by

$$
\boldsymbol{H}_{e f f}=\boldsymbol{H}_{s}-K P\left(\boldsymbol{M}_{h}\right) .
$$

Next we analyze the two terms on the right hand side of (25). First we use the fact that $P\left(M_{h}\right)$ is a projection to write

$$
\begin{aligned}
\left(\frac{\partial}{\partial t} \boldsymbol{M}_{h}, \boldsymbol{H}_{e f f}\right)_{h} & =\left(\frac{\partial}{\partial t} \boldsymbol{M}_{h}, \boldsymbol{H}_{s}\right)_{h}-K\left(\frac{\partial}{\partial t} \boldsymbol{M}_{h}, P\left(\boldsymbol{M}_{h}\right)\right)_{h} \\
& =\left(\frac{\partial}{\partial t} \boldsymbol{M}_{h}, \boldsymbol{H}_{s}\right)_{h}-K\left(\frac{\partial}{\partial t} P\left(\boldsymbol{M}_{h}\right), P\left(\boldsymbol{M}_{h}\right)\right)_{h} .
\end{aligned}
$$

Next, using the fact that $\boldsymbol{H}_{s}$ is static and $\boldsymbol{M}_{h}$ has time independent discrete norm, we can write

$$
\left(\frac{\partial}{\partial t} \boldsymbol{M}_{h}, \boldsymbol{H}_{e f f}\right)_{h}=-\frac{1}{2} \frac{d}{d t}\left(\left.\left\|\boldsymbol{M}_{h}-\boldsymbol{H}_{s}\right\|\right|^{2}+K\left\||| P\left(\boldsymbol{M}_{h}\right)\right\|^{2}\right) .
$$

To analyze the first term on the right hand side of (25), we choose $\boldsymbol{\xi}_{h}$ to be the interpolant of $\boldsymbol{H}_{T}$ in $(21)$

$$
\left(\frac{\partial}{\partial t} \boldsymbol{M}_{h}, \boldsymbol{H}_{T}\right)_{h}=-\frac{\alpha \gamma}{1+\alpha^{2}}\left(\frac{\boldsymbol{M}_{h}}{\left|\boldsymbol{M}_{h}\right|} \times\left(\boldsymbol{H}_{T} \times \boldsymbol{M}_{h}\right), \boldsymbol{H}_{T}\right)_{h} .
$$

Since the discrete inner product is computed pointwise we can rearrange the right hand side of the above expression and hence we deduce that

$$
\begin{aligned}
& \frac{1}{2} \frac{d}{d t}\left[\left\|\boldsymbol{E}_{h}(t)\right\|^{2}+\left\|\boldsymbol{H}_{h}(t)\right\|^{2}+\left\|\left|\left(\boldsymbol{H}_{s}-\boldsymbol{M}_{h}\right)(t)\|\|^{2}+K\|\mid\|\left(\boldsymbol{M}_{h}\right)(t)\|\|^{2}\right]\right.\right. \\
= & -\frac{\alpha \gamma}{1+\alpha^{2}}\left(\frac{\boldsymbol{H}_{T} \times \boldsymbol{M}_{h}}{\left|\boldsymbol{M}_{h}\right|}, \boldsymbol{H}_{T} \times \boldsymbol{M}_{h}\right)_{h} \leq 0 .
\end{aligned}
$$

and the result is proved.

\section{$5 \quad$ Error Estimates}

In this section we prove error estimates for the semi-discrete problem.

Let us define the error

$$
E(t)=\left\|\left(\boldsymbol{E}-\boldsymbol{E}_{h}\right)(t)\right\|^{2}+\left\|\left(\boldsymbol{H}-\boldsymbol{H}_{h}\right)(t)\right\|^{2}+\|\|\left(\boldsymbol{M}-\boldsymbol{M}_{h}\right)(t) \|^{2} .
$$

Also let $w_{h}:\left(L_{2}(\Omega)\right)^{3} \rightarrow V_{h}$ be the $\left(L_{2}(\Omega)\right)^{3}$ projection into $V_{h}$. The accuracy properties of the projection follow from the interpolation error estimate (24). Finally, recall that $\gamma^{\prime}=\frac{\gamma(1+\alpha)}{1+\alpha^{2}}$. We can then prove the following estimate:

Theorem 5.1 Let $T>0$ be the final time of integration for the solution. Let $A(T)$ be defined by

$\mathrm{RR} \mathrm{n}^{\circ} 3169$

$$
\begin{aligned}
A^{2}(T)=\max _{0 \leq s \leq T} & \left(\left\|\left(\boldsymbol{E}-r_{h} \boldsymbol{E}\right)(t)\right\|^{2}+\left\|\left(\boldsymbol{H}-w_{h} \boldsymbol{H}\right)(t)\right\|^{2}+\left\|\left(\boldsymbol{M}-w_{h} \boldsymbol{M}\right)(t)\right\|^{2}\right. \\
& +\left\|\boldsymbol{e}_{h}(0)\right\|^{2}+\left\|\boldsymbol{h}_{h}(0)\right\|^{2}+\left\|\boldsymbol{m}_{h}(0)\right\| \|^{2} \\
& +\int_{0}^{t} \delta\left(\left\|\left(r_{h} \boldsymbol{E}-\boldsymbol{E}\right)_{t}\right\|^{2}+\left\|r_{h} \boldsymbol{E}-\boldsymbol{E}\right\|^{2}+\left\|w_{h}^{Q} \boldsymbol{H}-\boldsymbol{H}\right\|^{2}\right. \\
& \left.\left.+\left\|\nabla \times\left(r_{h} \boldsymbol{E}-\boldsymbol{E}\right)\right\|^{2}+\left\|w_{h} \boldsymbol{H}-\boldsymbol{H}\right\|^{2}+\left\|\left(w_{h}^{Q} \boldsymbol{M}-\boldsymbol{M}\right)_{t}\right\|^{2}\right) d t\right)
\end{aligned}
$$


and suppose

$$
\mu_{T}=\max _{0 \leq t \leq T}\left(1+\frac{\alpha \gamma}{1+\alpha^{2}}\left(\left\|\boldsymbol{H}_{s}\right\|_{\infty, \Omega}+\|\boldsymbol{H}\|_{\infty, \Omega}+\left\|\boldsymbol{M}_{h}\right\|_{\infty, \Omega}+\|\boldsymbol{M}\|_{\infty, \Omega}\right)\right)<\infty,
$$

then for each $t$ with $0 \leq t \leq T$

$$
E(t) \leq A(T)^{2} \exp \left(C \mu_{T} t\right)
$$

Remarks: Assuming that the solution $(\boldsymbol{E}, \boldsymbol{H}, \boldsymbol{M})$ of the continuous problem is smooth enough on each element, we have the following estimate

$$
\left\|\left(\boldsymbol{E}-\boldsymbol{E}_{h}\right)(t)\right\|+\left\|\left(\boldsymbol{H}-\boldsymbol{H}_{h}\right)(t)\right\|+\|\|\left(\boldsymbol{M}-\boldsymbol{M}_{h}\right)(t)\|\|=O\left(h^{p}\right)
$$

where $p$ is the index in (23) and (24) and $0 \leq t \leq T$. The necessary smoothness of the solution comes from the norms in (23) and (24). We do not currently have the necessary a priori estimates to relate this smoothness to the data of the problem.

Before we start the proof, we define some notation. Let

$$
\boldsymbol{e}_{h}=r_{h} \boldsymbol{E}-\boldsymbol{E}_{h}, \quad \boldsymbol{h}_{h}=w_{h} \boldsymbol{H}-\boldsymbol{H}_{h}, \quad \boldsymbol{m}_{h}=w_{h}^{Q} \boldsymbol{M}-\boldsymbol{M}_{h}
$$

Proof. We start by expanding $\left(\boldsymbol{e}_{h}, \boldsymbol{e}_{h}\right)+\left(\boldsymbol{h}_{h}, \boldsymbol{h}_{h}\right)+\left(\boldsymbol{m}_{h}, \boldsymbol{m}_{h}\right)_{h}$ where the subscript $t$ denotes time differentiation:

$$
\begin{aligned}
\frac{1}{2} \frac{d}{d t}\left(\left(\boldsymbol{e}_{h}, \boldsymbol{e}_{h}\right)+\left(\boldsymbol{h}_{h}, \boldsymbol{h}_{h}\right)+\left(\boldsymbol{m}_{h}, \boldsymbol{m}_{h}\right)_{h}\right) \\
=\left(\boldsymbol{e}_{h, t}, \boldsymbol{e}_{h}\right)-\left(\boldsymbol{h}_{h}, \nabla \times \boldsymbol{e}_{h}\right)+\left(\boldsymbol{h}_{h, t}, \boldsymbol{h}_{h}\right)+\left(\nabla \times \boldsymbol{e}_{h}, \boldsymbol{h}_{h}\right)+\left(\boldsymbol{m}_{h, t}, \boldsymbol{m}_{h}\right) \\
=\quad\left(\left(r_{h} \boldsymbol{E}-\boldsymbol{E}\right)_{t}, \boldsymbol{e}_{h}\right)-\left(w_{h} \boldsymbol{H}-\boldsymbol{H}, \nabla \times \boldsymbol{e}_{h}\right)+\left(\left(\boldsymbol{E}-\boldsymbol{E}_{h}\right)_{t}, \boldsymbol{e}_{h}\right) \\
\quad-\left(\boldsymbol{H}-\boldsymbol{H}_{h}, \nabla \times \boldsymbol{e}_{h}\right)+\left(\left(w_{h} \boldsymbol{H}-\boldsymbol{H}\right)_{t}, \boldsymbol{h}_{h}\right)+\left(\nabla \times\left(r_{h} \boldsymbol{E}-\boldsymbol{E}\right), \boldsymbol{h}_{h}\right) \\
\quad+\left(\left(\boldsymbol{H}-\boldsymbol{H}_{h}\right)_{t}, \boldsymbol{h}_{h}\right)+\left(\nabla \times\left(\boldsymbol{E}-\boldsymbol{E}_{h}\right), \boldsymbol{h}_{h}\right)+\left(\left(w_{h}^{Q} \boldsymbol{M}-\boldsymbol{M}_{h}\right)_{t}, \boldsymbol{m}_{h}\right)_{h} .
\end{aligned}
$$

Now using the linearity of (20) and (21) and subtracting these equations from the corresponding equations for $\boldsymbol{E}$ and $\boldsymbol{H}$ we find that

$$
\begin{aligned}
\left(\left(\boldsymbol{E}-\boldsymbol{E}_{h}\right)_{t}, \boldsymbol{e}_{h}\right)-\left(\boldsymbol{H}-\boldsymbol{H}_{h}, \nabla \times \boldsymbol{e}_{h}\right) & =0 \\
\left(\left(\boldsymbol{H}-\boldsymbol{H}_{h}\right)_{t}, \boldsymbol{h}_{h}\right)+\left(\nabla \times\left(\boldsymbol{E}-\boldsymbol{E}_{h}\right), \boldsymbol{h}_{h}\right) & =-\left(\left(\boldsymbol{M}-\boldsymbol{M}_{h}\right)_{t}, \boldsymbol{h}_{h}\right)
\end{aligned}
$$

Furthermore, since we choose $w_{h}$ to be the $\left(L_{2}(\Omega)\right)^{3}$ projection onto $V_{h}$ and we assume that $\nabla \times U_{h} \subset V_{h}$ we can conclude that

$$
\left(w_{h} \boldsymbol{H}-\boldsymbol{H}, \nabla \times \boldsymbol{e}_{h}\right)=0, \text { and }\left(\left(w_{h} \boldsymbol{H}-\boldsymbol{H}\right)_{t}, \boldsymbol{h}_{h}\right)=0
$$

Hence, using these equalities and expanding the term $\left(\left(\boldsymbol{M}-\boldsymbol{M}_{h}\right)_{t}, \boldsymbol{h}_{h}\right)$ we arrive at the estimate

$$
\begin{aligned}
& \frac{1}{2} \frac{d}{d t}\left(\left(\boldsymbol{e}_{h}, \boldsymbol{e}_{h}\right)+\left(\boldsymbol{h}_{h}, \boldsymbol{h}_{h}\right)+\left(\boldsymbol{m}_{h}, \boldsymbol{m}_{h}\right)_{h}\right) \\
& =\quad\left(\left(r_{h} \boldsymbol{E}-\boldsymbol{E}\right)_{t}, \boldsymbol{e}_{h}\right)+\left(\nabla \times\left(r_{h} \boldsymbol{E}-\boldsymbol{E}_{h}\right), \boldsymbol{h}_{h}\right) \\
& \quad-\left(\left(\boldsymbol{M}-w_{h}^{Q} \boldsymbol{M}\right)_{t}, \boldsymbol{h}_{h}\right)-\left(\left(w_{h}^{Q} \boldsymbol{M}-\boldsymbol{M}_{h}\right)_{t}, \boldsymbol{h}_{h}\right) \\
& \quad+\left(\left(w_{h}^{Q} \boldsymbol{M}-\boldsymbol{M}_{h}\right)_{t}, \boldsymbol{m}_{h}\right)_{h} .
\end{aligned}
$$


Now we estimate the second two terms on the right hand side of (27). Using the equivalence of the discrete and continuous inner products on $V_{h}$ and (22) we obtain:

$$
\begin{aligned}
& \left(\left(w_{h}^{Q} \boldsymbol{M}-\boldsymbol{M}_{h}\right)_{t}, \boldsymbol{m}_{h}\right) \\
& \quad=\left(\left(w_{h}^{Q} \boldsymbol{M}-\boldsymbol{M}\right)_{t}, \boldsymbol{m}_{h}\right)_{h}+\left(\left(\boldsymbol{M}-\boldsymbol{M}_{h}\right)_{t}, \boldsymbol{m}_{h}\right)_{h} \\
& \quad=\left(F(\boldsymbol{H}, \boldsymbol{M})-F\left(\boldsymbol{H}_{h}, \boldsymbol{M}_{h}\right), w_{h}^{Q} \boldsymbol{M}-\boldsymbol{M}_{h}\right)_{h}
\end{aligned}
$$

where we have used the fact that $w_{h}^{Q}$ is the discrete projection to set $\left(\left(w_{h}^{Q} \boldsymbol{M}-\boldsymbol{M}\right)_{t}, \boldsymbol{m}_{h}\right)_{h}=0$. Hence, using Lemma 2.1, the Cauchy-Schwarz inequality and expanding terms

$$
\begin{aligned}
& \left|\left(\left(w_{h}^{Q} \boldsymbol{M}-\boldsymbol{M}_{h}\right)_{t}, \boldsymbol{m}_{h}\right)\right| \leq \\
& \leq \gamma^{\prime}\left(\left\|\boldsymbol{H}_{s}\right\|_{\infty, \Omega}+\|\boldsymbol{H}\|_{\infty, \Omega}+\left\|\boldsymbol{M}_{h}\right\|_{\infty, \Omega}\right. \\
& \left.+\|\boldsymbol{M}\|_{\infty, \Omega}\right)\left(\left\|\boldsymbol{M}-\boldsymbol{M}_{h}|\|+|\left|\boldsymbol{H}-\boldsymbol{H}_{h}\right|||\right)||\left|w_{h}^{Q} \boldsymbol{M}-\boldsymbol{M}_{h}\right| \|\right. \\
& \leq \gamma^{\prime}\left(\left\|\boldsymbol{H}_{s}\right\|_{\infty, \Omega}+\|\boldsymbol{H}\|_{\infty, \Omega}+\left\|\boldsymbol{M}_{h}\right\|_{\infty, \Omega}\right. \\
& \left.+\|\boldsymbol{M}\|_{\infty, \Omega}\right)\left(\left\|\boldsymbol{M}-\boldsymbol{M}_{h}\right\||+||| \boldsymbol{H}-w_{h}^{Q} \boldsymbol{H}\left\|\left|+\left\|\mid w_{h}^{Q} \boldsymbol{H}-\boldsymbol{H}_{h}\right\| \|\right)\right\||| w_{h}^{Q} \boldsymbol{M}-\boldsymbol{M}_{h}\|\|\right.
\end{aligned}
$$

Now using the equivalence of the discrete and continuous norms on $V_{h}$ and the arithmetic geometric mean inequality we obtain

$$
\begin{aligned}
& \left|\left(\left(w_{h}^{Q} \boldsymbol{M}-\boldsymbol{M}_{h}\right)_{t}, \boldsymbol{m}_{h}\right)\right| \leq \\
& \leq \quad C\left(\left\|\boldsymbol{H}_{s}\right\|_{\infty, \Omega}+\|\boldsymbol{H}\|_{\infty, \Omega}+\left\|\boldsymbol{M}_{h}\right\|_{\infty, \Omega}+\|\boldsymbol{M}\|_{\infty, \Omega}\right)\left(\left\|\boldsymbol{M}-\boldsymbol{M}_{h}\right\| \mid\right. \\
& \left.\quad+\left\|\boldsymbol{H}-w_{h}^{Q} \boldsymbol{H}\right\|\|+\| w_{h}^{Q} \boldsymbol{H}-\boldsymbol{H}\|+\| \boldsymbol{H}-\boldsymbol{H}_{h} \|\right)\left\|w_{h}^{Q} \boldsymbol{M}-\boldsymbol{M}_{h}\right\| \mid \\
& \leq C\left(\left\|\boldsymbol{H}_{s}\right\|_{\infty, \Omega}+\|\boldsymbol{H}\|_{\infty, \Omega}+\left\|\boldsymbol{M}_{h}\right\|_{\infty, \Omega}+\|\boldsymbol{M}\|_{\infty, \Omega}\right)\left(\left\|\boldsymbol{M}-\boldsymbol{M}_{h}\right\|^{2}\right. \\
& \left.\quad+\left\|w_{h}^{Q} \boldsymbol{H}-\boldsymbol{H}\right\|^{2}+\left\|\boldsymbol{H}-\boldsymbol{H}_{h}\right\|^{2}\right),
\end{aligned}
$$

where we have again used the fact that $\left\|\boldsymbol{H}-w_{h}^{Q} \boldsymbol{H}\right\| \mid=0$ and $\left\|\left|w_{h}^{Q} \boldsymbol{M}-\boldsymbol{M}_{h}\|\|=\left\|\mid \boldsymbol{M}-\boldsymbol{M}_{h}\right\| \|\right.\right.$.

We proceed in a similar way to estimate the term $\left(\left(w_{h}^{Q} \boldsymbol{M}-\boldsymbol{M}_{h}\right)_{t}, \boldsymbol{h}_{h}\right)$ :

$$
\begin{aligned}
& \left(\left(w_{h}^{Q} \boldsymbol{M}-\boldsymbol{M}_{h}\right)_{t}, \boldsymbol{h}_{h}\right)=\left(\left(w_{h}^{Q} \boldsymbol{M}-\boldsymbol{M}_{h}\right)_{t}, \boldsymbol{h}_{h}\right)_{h} \\
& \quad=\left(\left(\boldsymbol{M}-\boldsymbol{M}_{h}\right)_{t}, \boldsymbol{h}_{h}\right)_{h} \\
& \quad=\left(F(\boldsymbol{H}, \boldsymbol{M})-F\left(\boldsymbol{H}_{h}, \boldsymbol{M}_{h}\right), w_{h} \boldsymbol{H}-\boldsymbol{H}_{h}\right)_{h} .
\end{aligned}
$$

Now we expand terms and use the lemma, the Cauchy-Schwarz and arithmetic geometric mean inequalities to obtain:

$$
\begin{aligned}
& \left|\left(\left(w_{h}^{Q} \boldsymbol{M}-\boldsymbol{M}_{h}\right)_{t}, \boldsymbol{h}_{h}\right)\right| \leq C\left(\left\|\boldsymbol{H}_{s}\right\|_{\infty, \Omega}+\|\boldsymbol{H}\|_{\infty, \Omega}+\left\|\boldsymbol{M}_{h}\right\|_{\infty, \Omega}+\|\boldsymbol{M}\|_{\infty, \Omega}\right) \\
& \left(||\left|\boldsymbol{M}-\boldsymbol{M}_{h}\right|\left\|^{2}+\right\| \boldsymbol{H}-w_{h} \boldsymbol{H}\left\|^{2}+\right\| w_{h}^{Q} \boldsymbol{H}-\boldsymbol{H}\left\|^{2}+\right\| \boldsymbol{H}-\boldsymbol{H}_{h} \|^{2}\right) .
\end{aligned}
$$

Combining (28) and (29) in (27) and using the arithmetic-geometric mean inequality shows that

$$
\begin{aligned}
& \frac{d}{d t}\left(\left(\boldsymbol{e}_{h}, \boldsymbol{e}_{h}\right)+\left(\boldsymbol{h}_{h}, \boldsymbol{h}_{h}\right)+\left(\boldsymbol{m}_{h}, \boldsymbol{m}_{h}\right)_{h}\right) \\
& \leq C\left\{\begin{array}{l}
\left(\left\|\left(r_{h} \boldsymbol{E}-\boldsymbol{E}\right)_{t}\right\|^{2}+\left\|\boldsymbol{h}_{h}\right\|^{2}+\left\|\nabla \times\left(r_{h} \boldsymbol{E}-\boldsymbol{E}\right)\right\|^{2}+\left\|\boldsymbol{e}_{h}\right\|^{2}+\left\|\left(w_{h}^{Q} \boldsymbol{M}-\boldsymbol{M}\right)_{t}\right\|^{2}\right) \\
+\gamma^{\prime}\left(\left\|\boldsymbol{H}_{s}\right\|_{\infty, \Omega}+\|\boldsymbol{H}\|_{\infty, \Omega}+\left\|\boldsymbol{M}_{h}\right\|_{\infty, \Omega}+\|\boldsymbol{M}\|_{\infty, \Omega}\right)
\end{array}\right. \\
& \left.\quad\left(\left\|\boldsymbol{M}-\boldsymbol{M}_{h}\right\|^{2}+\left\|\boldsymbol{H}-\boldsymbol{H}_{h}\right\|^{2}+\left\|w_{h} \boldsymbol{H}-\boldsymbol{H}\right\|^{2}+\left\|w_{h}^{Q} \boldsymbol{H}-\boldsymbol{H}\right\|^{2}\right)\right\} .
\end{aligned}
$$

$\mathrm{RR} \mathrm{n}^{\circ} 3169$ 
Further expanding the terms $\left\|\boldsymbol{e}_{h}\right\|^{2}$ and $\left\|\boldsymbol{h}_{h}\right\|^{2}$ and collecting terms we obtain

$$
\begin{aligned}
& \frac{d}{d t}\left(\left(\boldsymbol{e}_{h}, \boldsymbol{e}_{h}\right)+\left(\boldsymbol{h}_{h}, \boldsymbol{h}_{h}\right)+\left(\boldsymbol{m}_{h}, \boldsymbol{m}_{h}\right)_{h}\right) \\
& \leq \delta\left(\left\|\left(r_{h} \boldsymbol{E}-\boldsymbol{E}\right)_{t}\right\|^{2}+\left\|r_{h} \boldsymbol{E}-\boldsymbol{E}\right\|^{2}+\left\|w_{h}^{Q} \boldsymbol{H}-\boldsymbol{H}\right\|^{2}\right. \\
&+\left\|\nabla \times\left(r_{h} \boldsymbol{E}-\boldsymbol{E}\right)\right\|^{2}+\left\|w_{h} \boldsymbol{H}-\boldsymbol{H}\right\|^{2}+\left\|\left(w_{h}^{Q} \boldsymbol{M}-\boldsymbol{M}\right)_{t}\right\|^{2} \\
&\left.+\left\|\boldsymbol{M}-\boldsymbol{M}_{h}\right\|\left\|^{2}+\right\| \boldsymbol{H}-\boldsymbol{H}_{h}\left\|^{2}+\right\| \boldsymbol{E}-\boldsymbol{E}_{h} \|^{2}\right) .
\end{aligned}
$$

where

$$
\delta=C\left(1+\frac{\alpha \gamma}{1+\alpha^{2}}\left(\left\|\boldsymbol{H}_{s}\right\|_{\infty, \Omega}+\|\boldsymbol{H}\|_{\infty, \Omega}+\left\|\boldsymbol{M}_{h}\right\|_{\infty, \Omega}+\|\boldsymbol{M}\|_{\infty, \Omega}\right)\right) .
$$

Integrating (30) we have shown that

$$
\begin{aligned}
& \left\|\left(\boldsymbol{E}-\boldsymbol{E}_{h}\right)(t)\right\|^{2}+\left\|\left(\boldsymbol{H}-\boldsymbol{H}_{h}\right)(t)\right\|^{2}+\left\|\left(\boldsymbol{M}-\boldsymbol{M}_{h}\right)(t)\right\| \|^{2} \\
& \leq\left\|\left(\boldsymbol{E}-r_{h} \boldsymbol{E}\right)(t)\right\|^{2}+\left\|\left(\boldsymbol{H}-w_{h} \boldsymbol{H}\right)(t)\right\|^{2}+\|\|\left(\boldsymbol{M}-w_{h} \boldsymbol{M}\right)(t) \|^{2} \\
& \quad+\left\|\boldsymbol{e}_{h}(t)\right\|^{2}+\left\|\boldsymbol{h}_{h}(t)\right\|^{2}+\left\|\boldsymbol{m}_{h}(t)\right\| \|^{2} \\
& \leq\left\|\left(\boldsymbol{E}-r_{h} \boldsymbol{E}\right)(t)\right\|^{2}+\left\|\left(\boldsymbol{H}-w_{h} \boldsymbol{H}\right)(t)\right\|^{2}+\left\|\mid\left(\boldsymbol{M}-w_{h} \boldsymbol{M}\right)(t)\right\| \|^{2} \\
& \quad+\left\|\boldsymbol{e}_{h}(0)\right\|^{2}+\left\|\boldsymbol{h}_{h}(0)\right\|^{2}+\left\|\boldsymbol{m}_{h}(0)\right\| \|^{2} \\
& +\int_{0}^{t} \delta\left(\left\|\left(r_{h} \boldsymbol{E}-\boldsymbol{E}\right)_{t}\right\|^{2}+\left\|r_{h} \boldsymbol{E}-\boldsymbol{E}\right\|^{2}+\left\|w_{h}^{Q} \boldsymbol{H}-\boldsymbol{H}\right\|^{2}\right. \\
& +\left\|\nabla \times\left(r_{h} \boldsymbol{E}-\boldsymbol{E}\right)\right\|^{2}+\left\|w_{h} \boldsymbol{H}-\boldsymbol{H}\right\|^{2}+\left\|\left(w_{h}^{Q} \boldsymbol{M}-\boldsymbol{M}\right)_{t}\right\|^{2} \\
& \left.+\left\|\boldsymbol{M}-\boldsymbol{M}_{h}\right\|\left\|^{2}+\right\| \boldsymbol{H}-\boldsymbol{H}_{h}\left\|^{2}+\right\| \boldsymbol{E}-\boldsymbol{E}_{h} \|^{2}\right) d t
\end{aligned}
$$

Now let $A(T)$ be defined by $(26)$ and let $\delta_{T}=\max _{0 \leq t \leq T} \delta$. For $0 \leq t \leq T$ we can rewrite (31) as

$$
\begin{aligned}
& \left\|\left(\boldsymbol{E}-\boldsymbol{E}_{h}\right)(t)\right\|^{2}+\left\|\left(\boldsymbol{H}-\boldsymbol{H}_{h}\right)(t)\right\|^{2}+\|\|\left(\boldsymbol{M}-\boldsymbol{M}_{h}\right)(t)\|\|^{2} \\
& \quad \leq A^{2}(T)+\delta_{T} \int_{0}^{t}\left(\left\|\boldsymbol{E}-\boldsymbol{E}_{h}\right\|^{2}+\left\|\boldsymbol{H}-\boldsymbol{H}_{h}\right\|^{2}+\left\|\boldsymbol{M}-\boldsymbol{M}_{h}\right\| \|^{2}\right) d t .
\end{aligned}
$$

Using the Gronwall inequality shows that

$$
\left\|\left(\boldsymbol{E}-\boldsymbol{E}_{h}\right)(t)\right\|^{2}+\left\|\left(\boldsymbol{H}-\boldsymbol{H}_{h}\right)(t)\right\|^{2}+\|\|\left(\boldsymbol{M}-\boldsymbol{M}_{h}\right)(t) \|^{2} \leq A(T)^{2} \exp \left(\delta_{T} t\right) .
$$

\section{Fully Discrete Scheme}

We choose an explicit/implicit time-stepping scheme that guarantees the conservation of the norm of $\boldsymbol{M}_{h}$ pointwise. In fact, if mass lumping is used, the scheme only requires a local system be solved at each interpolation point for $\boldsymbol{M}_{h}$ and hence the scheme can be time stepped rapidly.

Let $\Delta t>0$ be the time step. Then we wish to compute $\left(\boldsymbol{E}_{h}^{(n)}, \boldsymbol{H}_{h}^{(n+1 / 2)}, \boldsymbol{M}_{h}^{(n+1 / 2)}\right) \in$ $U_{h} \times V_{h} \times V_{h}$ for $n=0,1,, \cdots$ such that

$$
\boldsymbol{E}_{h}^{(n)} \approx \boldsymbol{E}_{h}\left(t_{n}\right), \quad \boldsymbol{H}_{h}^{(n+1 / 2)} \approx \boldsymbol{H}_{h}\left(t_{n+1 / 2}\right), \quad \boldsymbol{M}_{h}^{(n+1 / 2)} \approx \boldsymbol{M}_{h}\left(t_{n+1 / 2}\right),
$$


where $t_{n}=n \Delta t$. The initial data for $\boldsymbol{E}$ gives $\boldsymbol{E}_{h}^{(0)}$. The values of $\boldsymbol{H}_{h}^{(1 / 2)}$ and $\boldsymbol{M}_{h}^{(1 / 2)}$ can then be found by a half time step of a suitable explicit scheme (for example Runge-Kutta). From then on $\left(\boldsymbol{E}_{h}^{(n+1)}, \boldsymbol{H}_{h}^{(n+3 / 2)}, \boldsymbol{M}_{h}^{(n+3 / 2)}\right)$ is determined from $\left(\boldsymbol{E}_{h}^{(n)}, \boldsymbol{H}_{h}^{(n+1 / 2)}, \boldsymbol{M}_{h}^{(n+1 / 2)}\right)$ by

$$
\begin{aligned}
&\left(\frac{\boldsymbol{E}_{h}^{(n+1)}-\boldsymbol{E}_{h}^{(n)}}{\Delta t}, \boldsymbol{\psi}_{h}\right)-\left(\boldsymbol{H}_{h}^{(n+1 / 2)}, \nabla \times \boldsymbol{\psi}_{h}\right)=\left(\boldsymbol{J}\left(t_{n+1 / 2}\right), \boldsymbol{\psi}_{h}\right) \quad \forall \boldsymbol{\psi}_{h} \in U_{h} \\
&\left(\frac{\boldsymbol{H}_{h}^{(n+3 / 2)}-\boldsymbol{H}_{h}^{(n+1 / 2)}}{\Delta t}, \boldsymbol{\phi}_{h}\right)+\left(\nabla \times \boldsymbol{E}_{h}^{(n+1)}, \boldsymbol{\phi}_{h}\right)=-\left(\frac{\boldsymbol{M}_{h}^{(n+3 / 2)}-\boldsymbol{M}_{h}^{(n+1 / 2)}}{\Delta t}, \boldsymbol{\phi}_{h}\right) \\
&\left(\frac{\boldsymbol{M}_{h}^{(n+3 / 2)}-\boldsymbol{M}_{h}^{(n+1 / 2)}}{\Delta t}, \boldsymbol{\xi}_{h}\right)_{h}=\left(F\left(\boldsymbol{H}_{h}^{(n+1)}, \boldsymbol{M}_{h}^{(n+1)}\right), \boldsymbol{\xi}_{h}\right)_{h} \\
& \forall \boldsymbol{\xi}_{h} \in V_{h}
\end{aligned}
$$

where

$$
\boldsymbol{H}_{h}^{(n+1)}=\left(\boldsymbol{H}_{h}^{(n+3 / 2)}+\boldsymbol{H}_{h}^{(n+1 / 2)}\right) / 2 \text { and } \boldsymbol{M}_{h}^{(n+1)}=\left(\boldsymbol{M}_{h}^{(n+3 / 2)}+\boldsymbol{M}_{h}^{(n+1 / 2)}\right) / 2 .
$$

Remark: Equation (32) is exactly the equation arising in a standard finite element discretization of Maxwell's equations. If a consistent "mass matrix" is used, a linear problem must be solved at each time step. This can be done efficiently using the conjugate gradient method $[12]$.

Due to the use of the discrete inner product, equation (34) is satisfied at the quadrature points of the discrete inner product and hence at the interpolation points for $V_{h}$. In addition, since $\nabla \times U_{h} \subset V_{h}$, equation (33) is satisfied pointwise. Thus both equations can be solved at the interpolation points without coupling between interpolation points. Furthermore, using properties of the cross-product it is possible to derive explicit formulae for $\boldsymbol{H}_{h}^{(n+3 / 2)}$ and $\boldsymbol{M}_{h}^{(n+3 / 2)}$ [5]. So it is not necessary to use Newton's method at each time step.

Next we verify that the time stepping scheme satisfies the same conservation properties as the semi-discrete scheme.

Lemma 6.1 Let $\boldsymbol{a}_{i}$ be an interpolation point for $V_{h}$ then for $n \geq 0$,

$$
\left|\boldsymbol{M}_{h}^{(n+1 / 2)}\left(\boldsymbol{a}_{i}\right)\right|=\left|\boldsymbol{M}_{h}^{(1 / 2)}\left(\boldsymbol{a}_{i}\right)\right|
$$

Proof. Similarly to the proof of Lemma 4.1, we choose $\boldsymbol{\xi}_{h}$ in (34) to interpolate $\boldsymbol{M}_{h}^{(n+3 / 2)}+$ $\boldsymbol{M}_{h}^{(n+1 / 2)}$ at $\boldsymbol{a}_{i}$ and vanish at all other points. By the definition of $\boldsymbol{H}^{(n+1)}$ and $\boldsymbol{M}^{(n+1)}$ we conclude that

$$
\left(\boldsymbol{M}_{h}^{(n+3 / 2)}\left(\boldsymbol{a}_{i}\right)-\boldsymbol{M}_{h}^{(n+1 / 2)}\left(\boldsymbol{a}_{i}\right)\right) \cdot\left(\boldsymbol{M}_{h}^{(n+3 / 2)}\left(\boldsymbol{a}_{i}\right)+\boldsymbol{M}_{h}^{(n+1 / 2)}\left(\boldsymbol{a}_{i}\right)\right)=0 .
$$

Hence $\left|\boldsymbol{M}_{h}^{(n+3 / 2)}\left(\boldsymbol{a}_{i}\right)\right|=\left|\boldsymbol{M}_{h}^{(n+1 / 2)}\left(\boldsymbol{a}_{i}\right)\right|$, and the result follows.

Lemma 6.2 Suppose $\boldsymbol{J}=0$, then for each time step $N \geq 0$,

$$
\begin{aligned}
& \left\|\boldsymbol{E}_{h}^{(N)}\right\|^{2}+\left\|\boldsymbol{H}_{h}^{(N+1 / 2)}\right\|^{2}+\left\|\left|\left(\boldsymbol{H}_{s}-\boldsymbol{M}_{h}\right)^{(N+1 / 2)}\left\|\left.\right|^{2}+K\right\|\right|\left|P\left(\boldsymbol{M}_{h}^{(N+1 / 2)}\right) \|\right|^{2}\right. \\
\leq & \left\|\boldsymbol{E}_{h}^{(0)}\right\|^{2}+\left\|\boldsymbol{H}_{h}^{(1 / 2)}\right\|^{2}+\left\|\left|\left(\boldsymbol{H}_{s}-\boldsymbol{M}_{h}\right)^{(1 / 2)}\left\|^{2}+K\right\|\right|\right\| P\left(\boldsymbol{M}_{h}^{(1 / 2)}\right) \|\left.\right|^{2}
\end{aligned}
$$

$\mathrm{RR} \mathrm{n}^{\circ} 3169$ 
Proof. The proof is a fully discrete version of the proof of Lemma 4.2. Using $\boldsymbol{\psi}_{h}=$ $\boldsymbol{E}_{h}^{(n+1)}-\boldsymbol{E}_{h}^{(n+1)}$ in (32) and $\boldsymbol{\phi}_{h}=\boldsymbol{H}_{h}^{(n+3 / 2)}-\boldsymbol{H}_{h}^{(n+1 / 2)}$ in (33) and adding we obtain:

$$
\begin{aligned}
& \left(\left\|\boldsymbol{E}_{h}^{(n+1)}\right\|^{2}-\left\|\boldsymbol{E}_{h}^{(n)}\right\|^{2}\right)+\left(\left\|\boldsymbol{H}_{h}^{(n+3 / 2)}\right\|^{2}-\left\|\boldsymbol{H}_{h}^{(n+1 / 2)}\right\|^{2}\right) \\
& +\Delta t\left(\nabla \times \boldsymbol{E}_{h}^{(n+1)}, \boldsymbol{H}_{h}^{(n+3 / 2)}\right)-\Delta t\left(\nabla \times \boldsymbol{E}_{h}^{(n)}, \boldsymbol{H}_{h}^{(n+1 / 2)}\right) \\
= & -\left(\boldsymbol{M}_{h}^{(n+3 / 2)}-\boldsymbol{M}_{h}^{(n+1 / 2)}, \boldsymbol{H}_{h}^{(n+3 / 2)}+\boldsymbol{H}_{h}^{(n+1 / 2)}\right)_{h} .
\end{aligned}
$$

As in the proof of Lemma 4.2,

$$
\begin{aligned}
& \left(\boldsymbol{M}_{h}^{(n+3 / 2)}-\boldsymbol{M}_{h}^{(n+1 / 2)}, \boldsymbol{H}_{h}^{(n+3 / 2)}+\boldsymbol{H}_{h}^{(n+1 / 2)}\right)_{h} \\
= & \left(\boldsymbol{M}_{h}^{(n+3 / 2)}-\boldsymbol{M}_{h}^{(n+1 / 2)}, \boldsymbol{H}_{T, h}^{(n+3 / 2)}+\boldsymbol{H}_{T, h}^{(n+1 / 2)}\right)_{h} \\
& -\left(\boldsymbol{M}_{h}^{(n+3 / 2)}-\boldsymbol{M}_{h}^{(n+1 / 2)}, \boldsymbol{H}_{e f f, h}^{(n+3 / 2)}+\boldsymbol{H}_{e f f, h}^{(n+1 / 2)}\right)_{h}
\end{aligned}
$$

where

$$
\boldsymbol{H}_{T, h}^{(n+1 / 2)}=\boldsymbol{H}_{h}^{(n+1 / 2)}+\boldsymbol{H}_{e f f, h}^{(n+1 / 2)}, \text { and } \boldsymbol{H}_{e f f, h}^{(n+1 / 2)}=w_{h}^{Q} \boldsymbol{H}_{s}-w_{h}^{Q} K P\left(\boldsymbol{M}_{h}^{(n+1 / 2)}\right) .
$$

Note that $\boldsymbol{H}_{e f f, h}^{(n+1 / 2)} \in V_{h}$.

Now using the definition of $F$, we see that

$$
\left(\boldsymbol{M}_{h}^{(n+3 / 2)}-\boldsymbol{M}_{h}^{(n+1 / 2)}, \boldsymbol{H}_{T, h}^{(n+3 / 2)}+\boldsymbol{H}_{T, h}^{(n+1 / 2)}\right)_{h} \geq 0 .
$$

Furthermore, using the fact that $\boldsymbol{H}_{s}$ is static, and the fact that the norm of $\boldsymbol{M}_{h}^{n+1 / 2}$ is conserved at the interpolation points, we see that

$$
\begin{aligned}
& \left(\boldsymbol{M}_{h}^{(n+3 / 2)}-\boldsymbol{M}_{h}^{(n+1 / 2)}, \boldsymbol{H}_{e f f, h}^{(n+3 / 2)}+\boldsymbol{H}_{e f f, h}^{(n+1 / 2)}\right)_{h} \\
= & \frac{1}{2}\left(\left\|\boldsymbol{M}_{h}^{(n+3 / 2)}-\boldsymbol{H}_{s}\right\|^{2}-\left.\left\|\boldsymbol{M}_{h}^{(n+1 / 2)}-\boldsymbol{H}_{s}\right\|\right|^{2}\right) \\
& -\frac{K^{2}}{2}\left(\left\|P\left(\boldsymbol{M}_{h}^{(n+3 / 2)}\right)\right\|^{2}-\left.\left\|P\left(\boldsymbol{M}_{h}^{(n+1 / 2)}\right)\right\|\right|^{2}\right) .
\end{aligned}
$$

Thus (35) becomes

$$
\begin{aligned}
& \left\|\boldsymbol{E}_{h}^{(n+1)}\right\|^{2}+\left\|\boldsymbol{H}_{h}^{(n+3 / 2)}\right\|^{2}+\left\|\left|\boldsymbol{M}_{h}^{n+3 / 2}-\boldsymbol{H}_{s}\left\|\left.\right|^{2}+K^{2}\right\| P\left(\boldsymbol{M}_{h}^{n+3 / 2}\right)\|\|^{2}\right.\right. \\
\leq & \left\|\boldsymbol{E}_{h}^{(n)}\right\|^{2}+\left\|\boldsymbol{H}_{h}^{(n+1 / 2)}\right\|^{2}+\left\|\boldsymbol{M}_{h}^{n+1 / 2}-\boldsymbol{H}_{s}\right\|\left\|^{2}+K^{2}\right\| P\left(\boldsymbol{M}_{h}^{n+1 / 2}\right)\|\|^{2} \\
& +\frac{\Delta t}{2}\left(\nabla \times \boldsymbol{E}_{h}^{(n+1)}, \boldsymbol{H}_{h}^{(n+3 / 2)}\right)-\frac{\Delta t}{2}\left(\nabla \times \boldsymbol{E}_{h}^{(n)}, \boldsymbol{H}_{h}^{(n+1 / 2)}\right) .
\end{aligned}
$$

Adding this estimate over $n$ we obtain

$$
\begin{aligned}
& \left\|\boldsymbol{E}_{h}^{(N+1)}\right\|^{2}+\left\|\boldsymbol{H}_{h}^{(N+3 / 2)}\right\|^{2}+\left\|\boldsymbol{M}_{h}^{(N+3 / 2)}-\boldsymbol{H}_{s}\right\|\left\|^{2}+K^{2}\right\| P\left(\boldsymbol{M}_{h}^{(N+3 / 2)}\right)\|\|^{2} \\
& -\frac{\Delta t}{2}\left(\nabla \times \boldsymbol{E}_{h}^{(N+1)}, \boldsymbol{H}_{h}^{(N+3 / 2)}\right) \\
\leq & \left\|\boldsymbol{E}_{h}^{(0)}\right\|^{2}+\left\|\boldsymbol{H}_{h}^{(1 / 2)}\right\|^{2}+\left.\left\|\boldsymbol{M}_{h}^{(1 / 2)}-\boldsymbol{H}_{s}\right\|\right|^{2}+K^{2}\left\|P\left(\boldsymbol{M}_{h}^{(1 / 2)}\right)\right\| \|^{2} \\
& -\frac{\Delta t}{2}\left(\nabla \times \boldsymbol{E}_{h}^{(0)}, \boldsymbol{H}_{h}^{(1 / 2)}\right) .
\end{aligned}
$$


But using the assumed quasi-uniformity of the mesh

$$
\left|\left(\nabla \times \boldsymbol{E}_{h}^{(N+1)}, \boldsymbol{H}_{h}^{(N+3 / 2)}\right)\right| \leq\left\|\nabla \times \boldsymbol{E}_{h}^{(N+1)}\right\|\left\|\boldsymbol{H}_{h}^{(N+3 / 2)}\right\| \leq C \frac{1}{h}\left\|\boldsymbol{E}_{h}^{(N+1)}\right\|\left\|\boldsymbol{H}_{h}^{(N+3 / 2)}\right\| .
$$

Hence

$$
\begin{aligned}
& \left(\frac{1}{2}-C \frac{\Delta t}{4 h}\right)\left(\left\|\boldsymbol{E}_{h}^{(N+1)}\right\|^{2}+\left\|\boldsymbol{H}_{h}^{(N+3 / 2)}\right\|^{2}\right)+\left\|\left|\boldsymbol{M}_{h}^{(N+3 / 2)}-\boldsymbol{H}_{s}\left\|\left.\right|^{2}+K^{2}\right\|\right| P\left(\boldsymbol{M}_{h}^{(N+3 / 2)}\right)\right\| \|^{2} \\
\leq & \left(\frac{1}{2}-C \frac{\Delta t}{4 h}\right)\left(\left\|\boldsymbol{E}_{h}^{(0)}\right\|^{2}+\left\|\boldsymbol{H}_{h}^{(1 / 2)}\right\|^{2}\right)+\left.\left\|\boldsymbol{M}_{h}^{(1 / 2)}-\boldsymbol{H}_{s}\right\|\right|^{2}+K^{2}\left\|\mid P\left(\boldsymbol{M}_{h}^{(1 / 2)}\right)\right\| \|^{2} .
\end{aligned}
$$

If $\Delta t / h$ is chosen small enough that

$$
\frac{1}{2}-C \frac{\Delta t}{4 h}>0
$$

the method is stable. Note that the constant $C$ is independent of $h$ and $\Delta t$ but does depend on the shape of the tetrahedra in the mesh via the quasi-uniformity assumption.

\section{Numerical Results}

This is not the place for elaborate numerical results. Instead we show the results from a simple validation problem that reduces to a one dimensional model problem similar to that studied in [5]. This problem demonstrates some of the features that make ferromagnetic layers interesting.

The code uses the linear tetrahedral elements described in section 3.1.1. One major modification compared to the theory presented in the previous sections is that we approximate the exact inner product

$$
\left(\frac{\boldsymbol{E}_{h}^{(n+1)}-\boldsymbol{E}_{h}^{n+1}}{\Delta t}, \boldsymbol{\psi}_{h}\right)
$$

in (32) using element-wise quadrature in which the vertices of the tetrahedra are used as quadrature points. This has the effect of decoupling degrees of freedom for the electric field at different vertices. Thus $\boldsymbol{E}_{h}^{(n+1)}$ can be computed by solving a series of small matrix problems associated with each vertex. We term this "partial mass lumping". We expect, but have not proved, that this quadrature will preserve the order of convergence of the method (see [2] for a discussion of quadrature error effects on elliptic problems).

The domain $\Omega$ is shown in Figure 5. The solution is assumed to be periodic in the $x$ and $y$ directions with period $L_{x}$ and $L_{y}$ respectively. The incoming wave travels along the $z$ axis from the left and interacts with a ferromagnetic layer of length $L_{m}$ at the right of the domain (shaded). This problem reduces to a one dimensional problem. The incoming wave is

$$
\boldsymbol{E}^{i n c}=E_{0}\left(\begin{array}{c}
0 \\
1 \\
0
\end{array}\right) g(f(t-z / c))
$$

$\mathrm{RR} \mathrm{n}^{\circ} 3169$ 


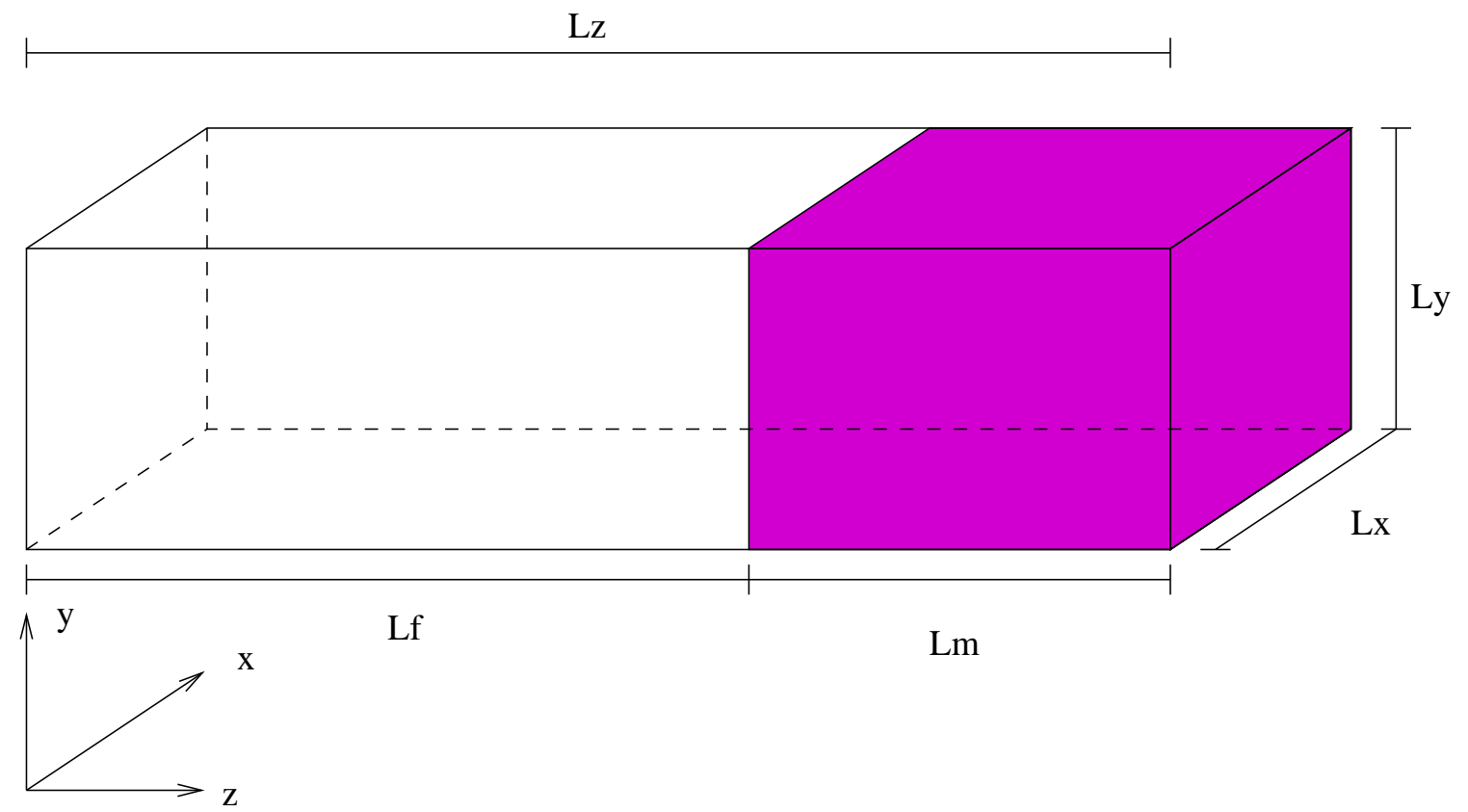

Figure 5: The domain for a periodic scatterer.

where $g$ is the Ricker pulse

$$
g(s)= \begin{cases}\frac{2 k}{11}\left(2 k(s-1.35)^{2}-1\right) \exp \left(-k(s-1.35)^{2}\right) & \text { if } t<3.48 \\ 0 & \text { if } t \geq 3.48\end{cases}
$$

and $k=(\pi / 1.31)^{2}$. We choose $E_{0}=100 c / \mu_{0}$. The values of $f, c$ and $\mu$ are given in Table 1 .

Incorporating periodicity into the computations is simple since the time stepping scheme is explicit. In addition we use inhomogeneous boundary conditions by interpolating boundary data. The effects of this interpolation also needs to be analyzed.

The initial state in the ferromagnet is

$$
\boldsymbol{M}=\left(\begin{array}{c}
0 \\
100 \\
0
\end{array}\right), \quad \boldsymbol{B}=\mu_{0} \boldsymbol{M} \text { and } \boldsymbol{E}=0 .
$$

Outside the ferromagnet, all the fields vanish. This choice of fields gives an initial field at equilibrium, and $\nabla \cdot \boldsymbol{B}=0$ at $t=0$. The parameters for our numerical experiments are given in Table 1.

We mesh the domain by dividing the $z$ dimension into $N_{z}=300$ subdivisions. This implies a $z$ mesh interval of $L_{z} / N_{z}$. We then choose $L_{x}=L_{y}=L_{z} / N_{z}$. The resulting prism is then divided into $N_{z}$ cubic cells. Each of these cells is then sub-divided into 6 tetrahedra to form the three dimensional grid. This gives $h \approx 0.133 \mathrm{~m}$ and we take $\Delta t=0.5 \times 10^{-10} \mathrm{~s}$, with a final time of integration $T=3 \times 10^{-7}$. Results at three different times are shown in Figures 6 and 


\begin{tabular}{||l|l||l|l||}
\hline \hline Parameter & Value & Parameter & Value \\
\hline$L_{z}$ & $40 \mathrm{~m}$ & $L_{x}$ & $0.4 \mathrm{~m}$ \\
$L_{y}$ & $0.4 \mathrm{~m}$ & $L_{m}$ & $16 \mathrm{~m}$ \\
$L_{f}$ & $24 \mathrm{~m}$ & & \\
$c$ & $1.3963 \times 10^{-9} \mathrm{~F} / \mathrm{m}$ & $\mu_{0}$ & $1.25667 \times 10^{-6} \mathrm{H} / \mathrm{m}$ \\
$E_{0}$ & $3 \times 10^{8} \mathrm{~m} / \mathrm{s}$ & $f$ & $4 \times 10^{7} \mathrm{~Hz}$ \\
$\alpha$ & $100 \mathrm{~A}$ & $\gamma$ & $2.2 \times 10^{5} \mathrm{~Hz} \mathrm{~m} / \mathrm{A}$ \\
\hline \hline
\end{tabular}

Table 1: Parameters for the one dimensional comparison problem

7. The incident field has vanishing $x$ and $z$ component, but after transiting the ferromagnetic layer the signal is "rotated" to make the $x$ component non-zero. In addition, since $\alpha \neq 0$ the layer is absorbing. Thus the magnitude of the reflected wave is also diminished.

The results computed in Figures 6 and 7 are computed with $N_{z}=300$. Similar results can be obtained using a one dimensional finite difference code with $N_{z}=100$. This is not surprising in view of the fact that the grid obtained by subdividing cubes into 6 tetrahedra is particularly poor for edge elements (strictly this is only demonstrated for non mass lumped edge elements of the first type [12] but the same problem is likely to afflict the element used here). Using a grid of cubes subdivided into five tetrahedra might improve the computation. Obviously the used of elements with better dispersion properties would improve the efficiency of the algorithm, and we are pursuing this approach now.

\section{Conclusion.}

We have presented a convergence theory for finite element methods for the Maxwell-LLG equations that includes a variety of elements. The resulting discrete equations have conservation properties similar to the continuous system, and this allows us to prove the error estimates.

Numerical results show that the linear tetrahedral scheme can be applied in three dimensions, but also reveals that the method can be expensive on certain grids. We plan to test higher order approaches in the future, and refine the error analysis to include mass-lumping and inhomogeneous boundary conditions. 

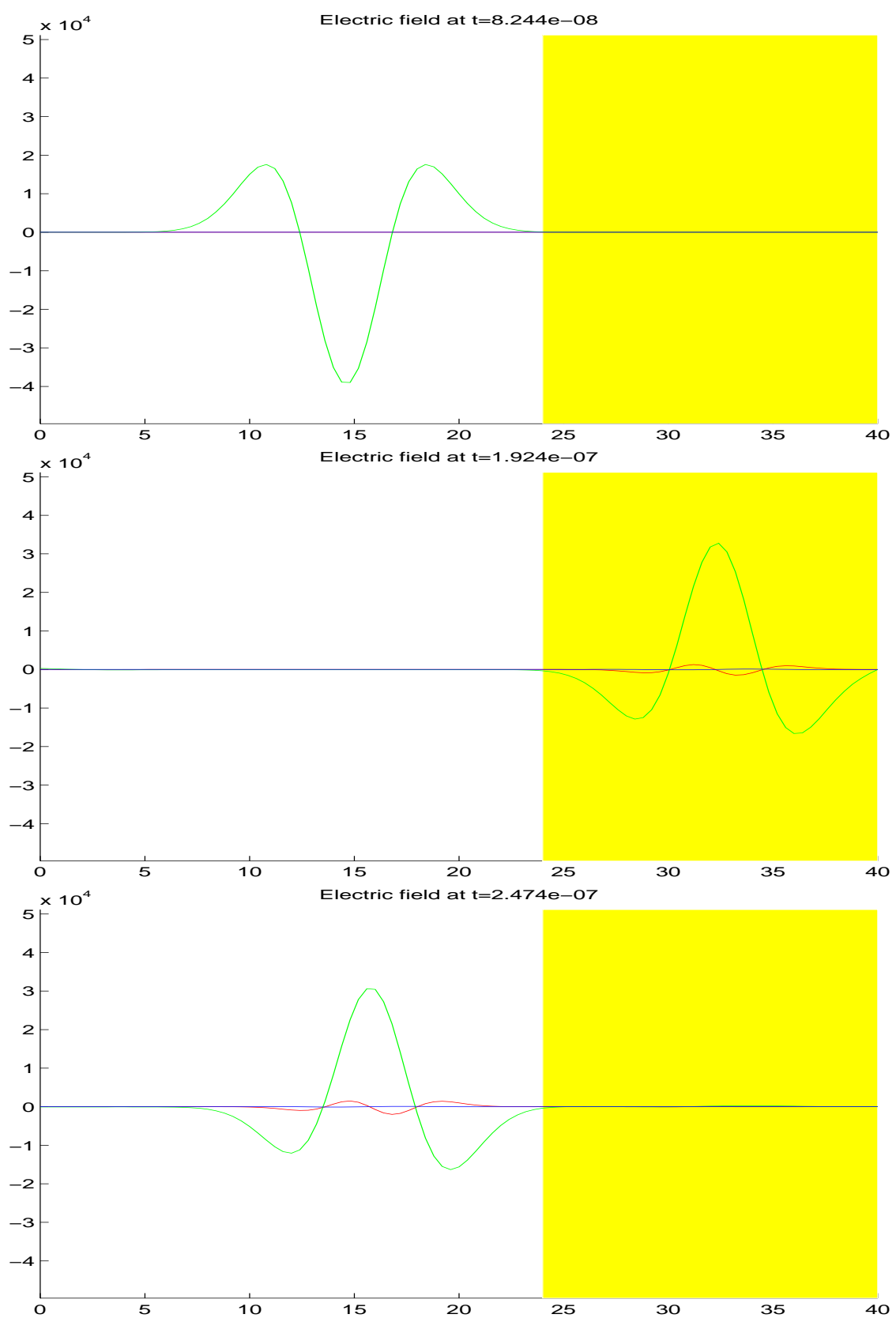

Figure 6: Here we show the electric field along the center line of the domain at three times. In the top panel, the incident wave is travelling to the right about to enter the ferromagnet (shaded). Only the $y$ component is visible. In the middle panel the wave has been reflected by the right wall. The ferromagnet has "rotated" the wave and the $x$ (small amplitude) and $y$ components are visible. In the bottom panel the wave has exited the ferromagnet. It is now lower amplitude and rotated. 

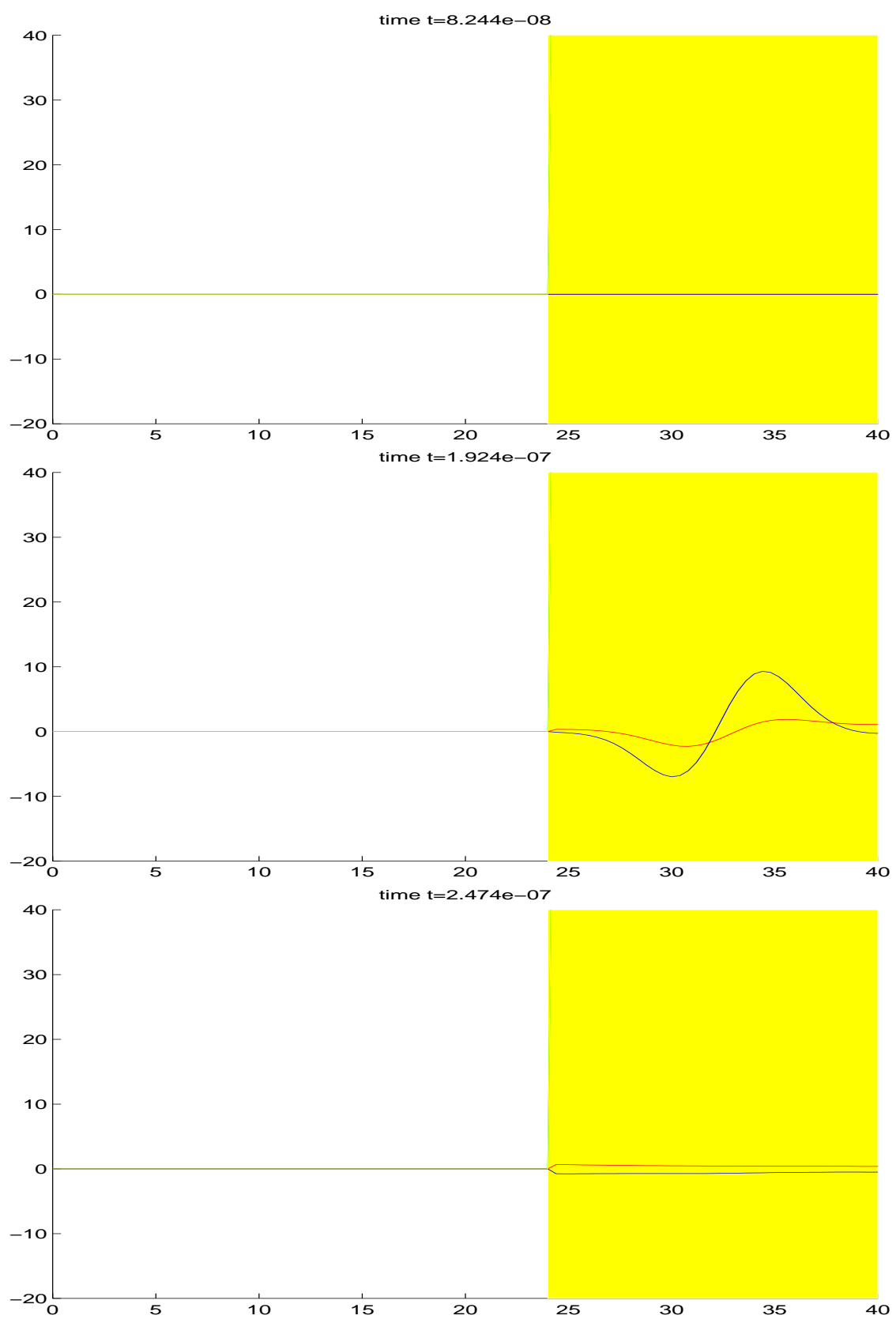

Figure 7: Here we show the $x$ and $z$ components of the magnetisation $\boldsymbol{M}$ along the center line of the domain at three times corresponding to the results in Figure 6 . In the top panel, the incident wave has not entered the ferromagnet so the magnetisation is at the initial state. In the middle panel the wave has been reflected by the right wall. The induced magnetisation is now visible (the larger amplitude wave is the $z$ component). In the bottom panel the wave has exited the ferromagnet. 


\section{Acknowledgment and Disclaimer}

Effort of Peter Monk sponsored by the Air Force Office of Scientific Research, Air Force Materials Command, USAF, under grant number F49620-95-1-0067. The US Government is authorized to reproduce and distribute reprints for governmental purposes notwithstanding any copyright notation thereon. The views and conclusions contained herein are those of the authors and should not be interpreted as necessarily representing the official policies or endorsements, either expressed or implied, of the Air Force Office of Scientific Research or the US Government.

This work was carried out while Peter Monk was visiting Project ONDES at INRIA, France.

\section{A A geometrical lemma}

In this appendix we prove the following identity :

$$
\begin{aligned}
& \forall\left(\mathbf{M}_{1}, \mathbf{M}_{2}, \mathbf{H}\right) \in\left(\mathbb{R}^{3}\right)^{3} \\
& \quad \Delta\left(\mathbf{M}_{1}, \mathbf{M}_{2}, \mathbf{H}\right)=\left|\frac{\mathbf{M}_{1}}{\left|\mathbf{M}_{1}\right|} \times\left(\mathbf{H} \times \mathbf{M}_{1}\right)-\frac{\mathbf{M}_{2}}{\left|\mathbf{M}_{2}\right|} \times\left(\mathbf{H} \times \mathbf{M}_{2}\right)\right| \leq|\mathbf{H}|\left|\mathbf{M}_{1}-\mathbf{M}_{2}\right|
\end{aligned}
$$

We decompose

$$
\mathbf{H}=\mathbf{H}_{\|}+\mathbf{H}_{\perp}
$$

where $\mathbf{H}_{\|}$is in the plane $\Pi$ defined by $\mathbf{M}_{1}$ and $\mathbf{M}_{2}$, while $\mathbf{H}_{\perp}$ is perpendicular to this plane. (Of course we assume $\frac{\mathbf{M}_{1}}{\left|\mathbf{M}_{1}\right|} \neq \frac{\mathbf{M}_{2}}{\left|\mathbf{M}_{2}\right|}$, otherwise the result is obvious.)

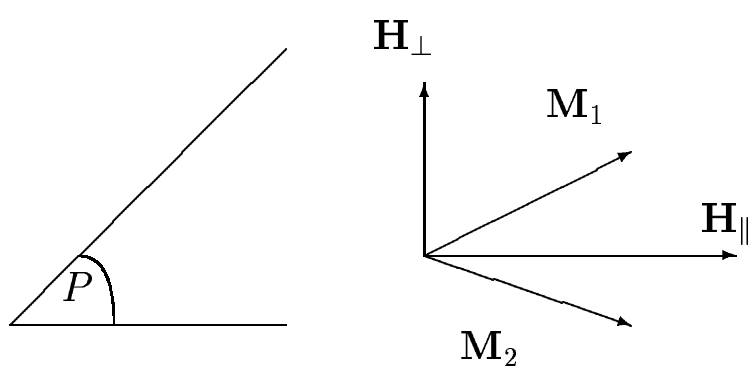

Note that $|\mathbf{H}|^{2}=\left|\mathbf{H}_{\|}\right|^{2}+\left|\mathbf{H}_{\perp}\right|^{2}$, and that

$$
\mathbf{M}_{i} \times\left(\mathbf{H}_{\|} \times \mathbf{M}_{i}\right) \in \Pi
$$

and

$$
\mathbf{M}_{i} \times\left(\mathbf{H}_{\perp} \times \mathbf{M}_{i}\right) \perp \Pi
$$

Thus we have, via Pythagorus's theorem,

$$
\begin{aligned}
\left|\Delta\left(\mathbf{M}_{1}, \mathbf{M}_{2}, \mathbf{H}\right)\right|^{2}=\mid \frac{\mathbf{M}_{1}}{\left|\mathbf{M}_{1}\right|} \times\left(\mathbf{H}_{\|} \times \mathbf{M}_{1}\right) & -\frac{\mathbf{M}_{2}}{\left|\mathbf{M}_{2}\right|} \times\left(\mathbf{H}_{\|} \times \mathbf{M}_{2}\right) \\
& +\frac{\mathbf{M}_{1}}{\left|\mathbf{M}_{1}\right|} \times\left(\mathbf{H}_{\perp} \times \mathbf{M}_{1}\right)-\frac{\mathbf{M}_{2}}{\left|\mathbf{M}_{2}\right|} \times\left.\left(\mathbf{H}_{\perp} \times \mathbf{M}_{2}\right)\right|_{\text {INRIA }} ^{2}
\end{aligned}
$$




$$
\begin{aligned}
=\mid \frac{\mathbf{M}_{1}}{\left|\mathbf{M}_{1}\right|} \times\left(\mathbf{H}_{\|} \times \mathbf{M}_{1}\right) & -\frac{\mathbf{M}_{2}}{\left|\mathbf{M}_{2}\right|} \times\left.\left(\mathbf{H}_{\|} \times \mathbf{M}_{2}\right)\right|^{2} \\
& +\left|\frac{\mathbf{M}_{1}}{\left|\mathbf{M}_{1}\right|} \times\left(\mathbf{H}_{\perp} \times \mathbf{M}_{1}\right)-\frac{\mathbf{M}_{2}}{\left|\mathbf{M}_{2}\right|} \times\left(\mathbf{H}_{\perp} \times \mathbf{M}_{2}\right)\right|^{2}
\end{aligned}
$$

As $\mathbf{H}_{\perp}$ is perpendicular to $\mathbf{M}_{1}$ and $\mathbf{M}_{2}$, we have

$$
\frac{\mathbf{M}_{i}}{\left|\mathbf{M}_{i}\right|} \times\left(\mathbf{H}_{\perp} \times \mathbf{M}_{i}\right)=\left|\mathbf{M}_{i}\right| \mathbf{H}_{\perp}
$$

which allows us to say that

$$
\begin{aligned}
\left|\frac{\mathbf{M}_{1}}{\left|\mathbf{M}_{1}\right|} \times\left(\mathbf{H}_{\perp} \times \mathbf{M}_{1}\right)-\frac{\mathbf{M}_{2}}{\left|\mathbf{M}_{2}\right|} \times\left(\mathbf{H}_{\perp} \times \mathbf{M}_{2}\right)\right|^{2} & =\left|\left(\left|\mathbf{M}_{1}\right|-\left|\mathbf{M}_{2}\right|\right) \mathbf{H}_{\perp}\right|^{2} \\
& =\left|\mathbf{H}_{\perp}\right|^{2}\left(\left|\mathbf{M}_{1}\right|-\left|\mathbf{M}_{2}\right|\right)^{2}
\end{aligned}
$$

and to conclude that

$$
\left|\frac{\mathbf{M}_{1}}{\left|\mathbf{M}_{1}\right|} \times\left(\mathbf{H}_{\perp} \times \mathbf{M}_{1}\right)-\frac{\mathbf{M}_{2}}{\left|\mathbf{M}_{2}\right|} \times\left(\mathbf{H}_{\perp} \times \mathbf{M}_{2}\right)\right|^{2} \leq\left|\mathbf{H}_{\perp}\right|^{2}\left|\mathbf{M}_{1}-\mathbf{M}_{2}\right|^{2}
$$

On the other hand, concerning $\mathbf{H}_{\|}$, we have

$$
\frac{\mathbf{H}_{\|} \times \mathbf{M}_{i}}{\left|\mathbf{H}_{\|}\right|\left|\mathbf{M}_{i}\right| \sin \theta_{i}} \times\left[\frac{\mathbf{M}_{i}}{\left|\mathbf{M}_{i}\right|} \times\left(\mathbf{H}_{\|} \times \mathbf{M}_{i}\right)\right]=\sin \theta_{i}\left|\mathbf{H}_{\|}\right| \mathbf{M}_{i}
$$

where

$$
\begin{aligned}
& \theta_{1}=\left({\widehat{\mathbf{H}} \|, \mathbf{M}_{1}}\right) \neq 0 \\
& \theta_{2}=\left({\widehat{\mathbf{H}} \|, \mathbf{M}_{2}}\right) \neq 0 \\
& \theta=\left({\widehat{\mathbf{M}}, \mathbf{M}_{2}}\right)=\theta_{2}-\theta_{1}
\end{aligned}
$$

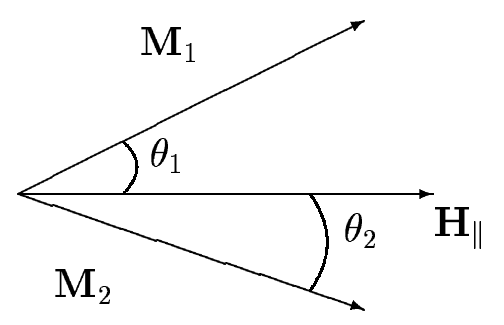

Since

$$
\frac{\mathbf{H}_{\|} \times \mathbf{M}_{1}}{\left|\mathbf{H}_{\|}\right|\left|\mathbf{M}_{1}\right| \sin \theta_{1}}=\frac{\mathbf{H}_{\|} \times \mathbf{M}_{2}}{\left|\mathbf{H}_{\|}\right|\left|\mathbf{M}_{2}\right| \sin \theta_{2}}=\mathbf{u}
$$

the crossproduct by this vector $\mathbf{u}$ defines a rotation - that is to say an isometry - which maps $\frac{\mathbf{M}_{i}}{\left|\mathbf{M}_{i}\right|} \times\left(\mathbf{H}_{\|} \times \mathbf{M}_{i}\right)$ on $\sin \theta_{i}\left|\mathbf{H}_{\|}\right| \mathbf{M}_{i}$, and thus we have

$$
\left|\frac{\mathbf{M}_{1}}{\left|\mathbf{M}_{1}\right|} \times\left(\mathbf{H}_{\|} \times \mathbf{M}_{1}\right)-\frac{\mathbf{M}_{2}}{\left|\mathbf{M}_{2}\right|} \times\left(\mathbf{H}_{\|} \times \mathbf{M}_{2}\right)\right|^{2}=\left|\mathbf{H}_{\|}\right|^{2}\left|\sin \theta_{1} \mathbf{M}_{1}-\sin \theta_{2} \mathbf{M}_{2}\right|^{2}
$$

It is then straightforward to compute that $\left|\mathbf{M}_{1}-\mathbf{M}_{2}\right|^{2}-\left|\sin \theta_{1} \mathbf{M}_{1}-\sin \theta_{2} \mathbf{M}_{2}\right|^{2}$ is equal to

$$
\begin{aligned}
= & \left|\mathbf{M}_{1}\right|^{2}+\left|\mathbf{M}_{2}\right|^{2}-2 \cos \theta\left|\mathbf{M}_{1}\right|\left|\mathbf{M}_{2}\right| \\
& \quad-\left(\sin ^{2} \theta_{1}\left|\mathbf{M}_{1}\right|^{2}+\sin ^{2} \theta_{2}\left|\mathbf{M}_{2}\right|^{2}-2 \sin \theta_{1} \sin \theta_{2}\left|\mathbf{M}_{1}\right|\left|\mathbf{M}_{2}\right|\right) \\
= & \cos ^{2} \theta_{1}\left|\mathbf{M}_{1}\right|^{2}+\cos ^{2} \theta_{2}\left|\mathbf{M}_{2}\right|^{2}-2 \cos \theta_{1} \cos \theta_{2}\left|\mathbf{M}_{1}\right|\left|\mathbf{M}_{2}\right| \\
= & \left(\cos \theta_{1}\left|\mathbf{M}_{1}\right|-\cos \theta_{2}\left|\mathbf{M}_{2}\right|\right)^{2} \geq 0
\end{aligned}
$$

$\mathrm{RR} \mathrm{n}^{\circ} 3169$ 
which shows, with (38), that

$$
\left|\frac{\mathbf{M}_{1}}{\left|\mathbf{M}_{1}\right|} \times\left(\mathbf{H}_{\|} \times \mathbf{M}_{1}\right)-\frac{\mathbf{M}_{2}}{\left|\mathbf{M}_{2}\right|} \times\left(\mathbf{H}_{\|} \times \mathbf{M}_{2}\right)\right|^{2} \leq\left|\mathbf{H}_{\|}\right|^{2}\left|\mathbf{M}_{1}-\mathbf{M}_{2}\right|^{2}
$$

Taking into account that $|\mathbf{H}|^{2}=\left|\mathbf{H}_{\|}\right|^{2}+\left|\mathbf{H}_{\perp}\right|^{2}$, we get the result by summing (37) and (40). 


\section{References}

[1] W. Brown, Micromagnetics, Tracts of Physics, Interscience, 1963.

[2] P. Ciarlet, The Finite Element Method for Elliptic Problems, vol. 4 of Studies In Mathematics and It's Applications, Elsevier North-Holland, New York, 1978.

[3] N. Dib and L. Katehi, Dispersion analysis of multilayer planar lines containing ferrite regions using an extended 2D-FDTD method, IEEE, (1993), pp. 842-844.

[4] F. Dubois, Discrete vector potential representation of a divergence free vector field in three dimensional domains: Numerical analysis of a model problem, SIAM J. Numer. Anal., 27 (1990), pp. 1103-1142.

[5] P. Joly and O. Vacus, Mathematical and numerical studies of $1 D$ non linear ferromagnetic materials, Tech. Report RR-3024, INRIA, 1996.

[6] P. Joly And O. Vacus, Mathematical and numerical studies of non linear ferromagnetic materials, in Numerical methods in engineering, ECCOMAS (Paris), 1996, pp. 325-331.

[7] P. Joly and O. Vacus, Maxwell's equations in a 1D ferromagnetic medium: existence and uniqueness of strong solutions, Tech. Report RR-3052, INRIA, 1996.

[8] B. Lax and K. Button, Microwave ferrites and ferrimagnetics, McGraw-Hill, 1962.

[9] J. Lee And R. Mittra, Analysis of microwave ferrite devices by using the finite element method, Journal of Applied Physics, 69 (1991), pp. 5032-5034.

[10] T. Monediere, M. Latrach, and F. Jecko, Resonant modes and magnetic losses in a ferrite coaxial resonator, Journal of Electromagnetics Waves and Applications, 6 (1992), pp. 199-217.

[11] P. Monk, An analysis of Nédélec's method for the spatial discretization of Maxwell's equations, J. Comp. Appl. Math., 47 (1993), pp. 101-121.

[12] P. Monk And A. Parrott, Analysis of finite element time domain methods in electromagnetic scattering. Oxford University Computing Lab. Numerical Analysis Report $96 / 25$.

[13] G. Mur And A. DE Hoop, A finite-element method for computing three-dimensional electromagnetic fields in inhomogeneous media, IEEE Trans. Magnetics, MAG-21 (1985), pp. $2188-2191$.

[14] J. NÉDÉLEC, Mixed finite elements in $\mathbb{R}^{3}$, Numer. Math., 35 (1980), pp. 315-341.

$[15]$ _ A new family of mixed finite elements in $\mathbb{R}^{3}$, Numer. Math., 50 (1986), pp. 57-81.

[16] J. Pereda, L. Vielva, M. Solano, A. Vega, and A. Prieto, FDtD analysis of magnetized ferritesl application to the calculation of dispersion characteristics of ferriteloaded waveguides, IEEE Transactions on Microwave Theorey and Techniques, 43 (1995), pp. 350-356.

$\mathrm{RR} \mathrm{n}^{\circ} 3169$ 
[17] J. Pereda, L. Vielva, A. Vegas, and A. Prieto, A treatment of magnetized ferrites using the FDTD method, IEEE Microwave and Guided Wave Letters, (1993), pp. 136-138.

[18] A. Reinex, T. Monediere, And F. JeCKo, Ferrite analysis using the finite-difference time domain method, IEEE Microwave and Optical Technology Letters, 5 (1992), pp. 685686.

[19] R. Silvester And R. Ferrari, Finite element methods for electrical engineers, Cambridge University Press, 1996.

[20] A. Visintin, On Landau-Lifchitz' equations for ferromagnetism, Japan journal of applied mathematics, 2 (1985), pp. 69-84.

[21] G. Zheng AND K. Chen, Nonlinear study of microstrip lines containing ferrite dielectric layers, Internal Journal of Infrared and Millimeter Waves, 13 (1992), pp. 1115-1125. 
Unit’e de recherche INRIA Lorraine, Technopôle de Nancy-Brabois, Campus scientifique, 615 rue du Jardin Botanique, BP 101, 54600 VILLERS LÈS NANCY

Unit'e de recherche INRIA Rennes, Irisa, Campus universitaire de Beaulieu, 35042 RENNES Cedex

Unit'e de recherche INRIA Rhône-Alpes, 655, avenue de l'Europe, 38330 MONTBONNOT ST MARTIN

Unit ${ }^{\prime}$ e de recherche INRIA Rocquencourt, Domaine de Voluceau, Rocquencourt, BP 105, 78153 LE CHESNAY Cedex

Unit e de recherche INRIA Sophia-Antipolis, 2004 route des Lucioles, BP 93, 06902 SOPHIA-ANTIPOLIS Cedex

\section{Éditeur}

INRIA, Domaine de Voluceau, Rocquencourt, BP 105, 78153 LE CHESNAY Cedex (France)

http://www.inria.fr

ISSN 0249-6399 